\title{
Hsp70/Bmi1-Fox01-SOD Signaling Pathway Contributes to the Protective Effect of Sound Conditioning against Acute Acoustic Trauma in a Rat Model
}

\author{
Guoxia Zhu, ${ }^{1,2}$ Yongxiang Wu, ${ }^{1,3,4}$ Yang Qiu, ${ }^{1}$ Keyong Tian $\mathbb{D}^{1},{ }^{1}$ Wenjuan Mi, ${ }^{1}$ Xinqin Liu, ${ }^{5}$ \\ Yuanyuan Chen, ${ }^{4}$ Jinwen Jia, ${ }^{6}$ Jiasheng Luo ${ }^{D},{ }^{2}$ Lianjun Lu $\mathbb{D}^{7},{ }^{7}$ and Jianhua Qiu $\mathbb{D}^{1}$ \\ ${ }^{1}$ Department of Otolaryngology-Head and Neck Surgery, Xijing Hospital, Fourth Military Medical University, Xi'an, Shaanxi, China \\ 710032 \\ ${ }^{2}$ Department of Otolaryngology, Head and Neck Surgery, Xi'an People's Hospital/Xi'an Fourth Hospital, Xi'an, Shaanxi, China \\ 710043 \\ ${ }^{3}$ Department of Otolaryngology-Head and Neck Surgery, Chinese PLA General Hospital, Beijing, China 100853 \\ ${ }^{4}$ Department of Otolaryngology-Head and Neck Surgery, General Hospital of Xinjiang Military Region, Urumchi, Xinjiang, China \\ 830011 \\ ${ }^{5}$ Department of Occupational and Environmental Health, Ministry of Education Key Lab of Hazard Assessment and Control in \\ Special Operational Environment and Shaanxi Key Laboratory of Free Radical Biology and Medicine, Fourth Military \\ Medical University, Xi'an, Shaanxi, China 710032 \\ ${ }^{6}$ Department of Otolaryngology, Head and Neck Surgery, The Fifth Affiliated Hospital of Xinjing Medical University, Urumchi, \\ Xinjiang, China 830011 \\ ${ }^{7}$ Department of Otolaryngology-Head and Neck Surgery, Tangdu Hospital, Fourth Military Medical University, Xi'an, China 710038
}

Correspondence should be addressed to Jiasheng Luo; 1-js@163.com, Lianjun Lu; lulianj@fmmu.edu.cn, and Jianhua Qiu; qiujh@fmmu.edu.cn

Guoxia Zhu, Yongxiang Wu, and Yang Qiu contributed equally to this work.

Received 15 April 2020; Revised 27 July 2020; Accepted 5 August 2020; Published 5 October 2020

Academic Editor: Renjie Chai

Copyright (C) 2020 Guoxia Zhu et al. This is an open access article distributed under the Creative Commons Attribution License, which permits unrestricted use, distribution, and reproduction in any medium, provided the original work is properly cited.

Sound conditioning (SC) is defined as "toughening" to lower levels of sound over time, which reduces a subsequent noise-induced threshold shift. Although the protective effect of SC in mammals is generally understood, the exact mechanisms involved have not yet been elucidated. To confirm the protective effect of SC against noise exposure (NE) and the stress-related signaling pathway of its rescue, we observed target molecule changes caused by SC of low frequency prior to NE as well as histology analysis in vivo and verified the suggested mechanisms in SGNs in vitro. Further, we investigated the potential role of Hsp70 and Bmil in SC by targeting SOD1 and SOD2 which are regulated by the FoxO1 signaling pathway based on mitochondrial function and reactive oxygen species (ROS) levels. Finally, we sought to identify the possible molecular mechanisms associated with the beneficial effects of SC against noise-induced trauma. Data from the rat model were evaluated by western blot, immunofluorescence, and RT-PCR. The results revealed that SC upregulated Hsp70, Bmil, FoxO1, SOD1, and SOD2 expression in spiral ganglion neurons (SGNs). Moreover, the auditory brainstem responses (ABRs) and electron microscopy revealed that SC could protect against acute acoustic trauma (AAT) based on a significant reduction of hearing impairment and visible reduction in outer hair cell loss as well as ultrastructural changes in OHCs and SGNs. Collectively, these results suggested that the contribution of Bmil toward decreased sensitivity to noise-induced trauma following SC was triggered by Hsp70 induction and associated with enhancement of the antioxidant system and decreased mitochondrial superoxide accumulation. This contribution of Bmil was achieved by direct targeting of SOD1 and SOD2, which was regulated by FoxO1. Therefore, the Hsp70/Bmi1-FoxO1-SOD signaling pathway might contribute to the protective effect of SC against AAT in a rat model. 


\section{Introduction}

Sound conditioning (SC), known as noise-induced "toughening," is widely defined as acoustic stimulation at a low intensity for an extended period of time prior to an elevated noise exposure (NE), which reduces the permanent threshold shift caused by a high-intensity sound. Clinically, SC has the greatest potential for treating acute acoustic trauma (AAT), as there is currently no other effective therapy. While SC has been shown to have a protective effect in most mammals, the specific mechanisms involved have not yet been explicated. Currently, there are several hypotheses regarding this mechanism: (1) functional reconstruction of OHCs, (2) upregulation of heat shock proteins (Hsp), (3) upregulation of antioxidative enzymes, (4) increases in stress-dependent metabolic activity, (5) alteration of intracellular calcium concentration, (6) increases in blood flow in the cochlea, and (7) changes in lateral efferent functional activity $[1,2]$. A previous study suggested that prior to a loud noise, SC (a pure tone of $500 \mathrm{~Hz}$ ) could enhance the removal of stressinduced free radicals to protect hearing [3]. Then, another study confirmed that SC of the low frequency (a pure tone of $1 \mathrm{kHz}$ ) prior to a loud noise upregulated tyrosine hydroxylase in the lateral efferent to protect against acoustic trauma [4]. Furthermore, another work showed that the protective mechanism of hair cells during SC of low frequency (sound conditioning prior to a loud noise) was carried out through an increase in cellular cytoskeleton proteins and through the relief of intracellular calcium overloading caused by NE [5]. Moreover, recent research suggested that the beneficial mechanisms of SC which was prior to a loud noise initiate in the cochlea and eventually reach the central auditory system. This phenomenon might be in part related to an interplay between the calretinin and nitric oxide signaling pathways and increases in the cytosolic calcium buffering capacity induced by SC [6]. In addition, the research by Roy et al. indicated that SC was also protective against two classes of ototoxic drugs (aminoglycosides and cisplatin) [7]. Therefore, it has been widely accepted that preconditioning to sound, especially sound conditioning of low frequency prior to a loud noise (but not sound conditioning after acoustic trauma), is a well-documented strategy to provide protection against $\mathrm{AAT}$ and the underlying mechanisms behind the protective effect of SC largely might refer to cochlear tissue.

Interestingly, these data lend further support to the growing body of evidence that specific gene polymorphisms may influence the susceptibility of noise-induced hearing loss, such as Rs3735715 polymorphisms in the GRHL2 gene [8], as well as Rs208679 and rs769217 polymorphisms in the Catalase (CAT) gene [9]. Furthermore, animal research has revealed that homologous animals have different threshold shifts following exposure to noise of the same intensity levels [10]. Pouyatos et al. found that OHCs in a high-frequency area were heavily injured in guinea pigs after the octave band noise $(4 \mathrm{kHz}, 110 \mathrm{~dB}$, and 8 or $16 \mathrm{kHz}$ at $97 \mathrm{~dB})$ exposure and intervention with an antioxidant inhibitor, while the OHCs from a low-frequency area and the inner hair cells exhibited almost normal [11]. Moreover, exogenous hydrogen peroxide induced a more severe impairment to the OHCs in the high-frequency area than in the other locations $[12,13]$. However, antioxidants rescued OHCs in high-frequency areas with a small amount of glutathione, which increased their survival rate, which was generally lower than that of normal guinea pigs [14]. All of the above findings provide evidence that OHCs in high-frequency areas are vulnerable to ROS due to insufficient antioxidation [15]. Thus far, an imbalance in the redox state caused by oxidative stress has been confirmed to play a crucial role in the progress of acoustic trauma [16-18]. Conversely, it is technically and theoretically feasible to enhance the antioxidant system and increase endogenous antioxidant by sound conditioning of low frequency prior to a loud noise, while higher frequencies or even noise might result in hearing loss.

Moreover, regardless of the tissue or cell type, they have their own means of protection against stress-induced acoustic trauma. In most stress incidents such as hypoxia, oxidative stress, temperature shock, and heavy metal poisoning, Hsp70 plays a key role in maintaining protein homeostasis and the correction of protein folding to promote cell survival by directly unfolding misfolded proteins in an ATP-dependent fashion [19]. Conversely, it has been demonstrated that Bmil, a member of the polycomb group transcription factors, has a significant role in hair cell survival by regulating the redox balance and ROS levels $[20,21]$. However, it is still unknown whether both Hsp70 and Bmil are genuinely involved in the protective effect of SC against AAT or not.

Therefore, based on the ROS-induced acoustic trauma caused by NE and SC of low frequency prior to a loud noise, the purpose of our study was to investigate the protective effect of SC against AAT and to confirm the stress-related signaling pathway of its rescue. Given the integrity of auditory pathways and the importance of afferent nerves, we specifically focused on both the organ of Corti and the SGNs. Here, we found that SC of low frequency not only protected against AAT based on significant improvements in hearing threshold and an apparent reduction in $\mathrm{OHC}$ loss but also improved SGN survival following noise-induced stress response via the increasing amount of mitochondria, regulating mitochondrial function and decreasing ROS levels in rat SGNs, and we first have demonstrated a new theory on the protection of SC against AAT in which upregulation of Hsp70, Bmi1, FoxO1, SOD is involved. Lastly, we suggested that the Hsp70/Bmil-FoxO1-SOD signaling pathway might contribute to the enhancement of the antioxidant system and a reduction in ROS accumulation for the decreased sensitivity to noise-induced trauma after treatment with SC. Our study was exploratory, and these results from our work could help us easily understand how to protect against AAT by SC and its underlying mechanisms; clinically, these issues will provide a better preventive strategy for AAT.

\section{Materials and Methods}

2.1. Animals and Exposure. In this study, 108 healthy adult male Sprague-Dawley rats weighing 200-300 g with a normal Preyer's reflex were provided by the Laboratory Animal Center of the Fourth Military Medical University. The inclusion and exclusion criteria were predetermined that all the 
animals in this study should have a good hearing without hearing loss caused by tympanitis, drug, noise, genetic problem, and so on. All rats had been raised with sufficient food and water in a tranquil animal cage (the plastic box with railing cover) for 5 days prior to the test (the sound levels were 20-30 dB in each cage in which every four rats were housed).

They were randomly divided into the control group (Ctrl), sound conditioning group (SC), noise exposure group (NE), and sound conditioning plus noise exposure group (SC $+\mathrm{NE}$ ) by the simple randomization, and the initial number of animals used per group was twenty-seven (Table of random number was used in our study. Each rat had its own number based on its weight which ranged from 1 to 108 . Then, every rat was given a random three-digit sequence generated by the random number generator (table of random number) in sequence. Next, these random three-digit sequences which had been assigned to rats were arranged in descending order. Finally, the top 27 rats were matched with Ctrl, the next 27 rats were matched with SC, the last 27 rats were matched with SC+NE, and the rest were matched with NE.). Six animals were excluded based on the exclusion criteria (tympanitis) or died during experiments. One rat in Ctrl, two rats in NE, and three rats in SC+NE were replaced before killing of animals. To minimize animal suffering, an intraperitoneal (i.p.) injection of pentobarbital sodium $(30 \mathrm{mg} / \mathrm{kg})$ was used during experiments. The NE group was exposed to white noise at $115 \mathrm{~dB}$ SPL for 6 hours per day over two consecutive days with anesthesia to lower stress hormones which might affect the results of the study; in the SC group, all of the animals were exposed to a pure tone of $1 \mathrm{kHz}$ at $85 \mathrm{~dB}$ SPL for 24 hours based on Niu and Canlon's study protocol [4]; the Ctrl group was given a sham exposure; the animals in the SC+NE group were exposed to SC before $\mathrm{NE}$ and were allowed to rest for 3 hours between SC and NE with anesthesia. The exposure protocol has been described in previous publications from our department [22, 23] (Figure 1(a)). Briefly, exposure was conducted in a ventilated soundproof cabinet where the animals had ad libitum access to food and water except for the rats anesthetized for the noise exposure. A Radio Shack Super Tweeter (Tandy Corp, FT Worth, USA), which was located above the cages, generated a noise (white noise, $115 \mathrm{~dB} \mathrm{SPL}$ ) and a pure tone $(1 \mathrm{kHz}$ at $85 \mathrm{~dB} \mathrm{SPL})$ that was then amplified by a power amplifier (Yamaha, Japan) and delivered to a loudspeaker. The homogeneity of the sound field was confirmed by a sound level meter (Bruel and Kjaer, China) that was secured within the cabinet.

\subsection{Auditory Brainstem Response (ABR) Measurement. ABRs} to both the click stimuli and the pure tone frequencies in the soundproof chamber on the day before exposure and at approximately 24 hours after the last exposure were available to evaluate hearing alterations in the SD rats in the Ctrl, SC, $\mathrm{NE}$, and $\mathrm{SC}+\mathrm{NE}$ groups. The ABR experimenter was unaware of the animal's group during ABR measurement. Each animal was gently anesthetized with an intraperitoneal (i.p.) injection of pentobarbital sodium $(30 \mathrm{mg} / \mathrm{kg})$ and then was placed on an electric heating plate $\left(37.1-37.5^{\circ} \mathrm{C}\right)$ to maintain body temperature. The reference electrode was placed beneath the pinna of the test ear, with the ground electrode placed beneath the apex of the nose, and the active electrode was placed beneath the skin on the top of the head. All of the electrodes were subcutaneously placed at each site within $5 \mathrm{~min}$ of the administration of anesthesia. The ABR test started immediately after the needle electrode implantation. Each test ear received the stimulus signal at a repeating rate of 10/s generated through Intelligent Hearing Systems (Bio-Logic Systems, USA), and the stimulus signal was delivered through earphones with a 10 min interval between left and right ears. The signal intensity was decreased gradually by a $5 \mathrm{~dB}$ step until the visually discernible $\mathrm{ABR}$ waveform disappeared. The lowest sound level that caused this waveform was defined as the "threshold." Five repetitions of each threshold were presented. The waves were amplified ten times by Intelligent Hearing Systems. The highest sound level was less than $90 \mathrm{~dB}$ to avoid drastic acoustic trauma [23, 24].

\subsection{Scanning Electron Microscopy (SEM) and Transmission} Electron Microscopy (TEM). Cochlear sensory epithelia surface preparation and $\mathrm{OHC}$ count in animals from the Ctrl, $\mathrm{SC}, \mathrm{NE}$, and SC+NE groups (each group: $n=6$ from 3 animals) were carried out as described in a previous study from our department. Briefly, following ABR measurement, deeply anesthetized animals from each group were decapitated on the first day after exposure. The cochleae were removed immediately and gently perfused with $2.5 \%$ phosphate-buffered glutaraldehyde ( $\mathrm{pH} 7.4)$ through the open round window and the cochlear apex. The cochleae remained in the same solution overnight. The bony capsule was removed after washing with $0.1 \mathrm{M}$ phosphate-buffered saline (PBS). The spiral ligament and stria vascularis were removed under a dissecting microscope, and the Reissner's membrane was separated. The dissected specimens were rinsed with $0.1 \mathrm{M}$ PBS, then postfixed in $1 \%$ osmium tetroxide for 2 hours, and incubated in $2 \%$ tannic acid twice for $30 \mathrm{~min}$. The cochleae were dehydrated in a series of graded ethanol solutions and dried in a critical point dryer (Hitachi, Japan). The specimens were fixed on a metal stage, goldcoated in a sputter coater (Ion Sputter, Hitachi, Japan), and observed under SEM (Hitachi, Japan). The experimenter was unaware of the animal's group during the experimentation of $\mathrm{OHC}$ count. OHCs were counted by hand using five consecutive images from each slide. The principle of counting is such that if an $\mathrm{OHC}$ is not completely contained within the image, the cell was counted only if found at the top or left edge of the image. The missing hair cells and stereocilia were quantified along the entire basilar membrane. The percentage of missing OHCs in each row was calculated and compared among the four groups $[22,23]$. In addition, the apex turn of the basilar membrane was considered as the low-frequency area of 0 to 30 percent distance from the apex for the cochlea; the middle turn was the middle-frequency area of 30 to 60 percent distance from the apex, and the base turn was the high-frequency area of 60 to 100 percent distance from the apex. Scale in Figure 2(c) showed frequency and percent distance from the apex for rat cochlea according to Muller's study [25]. 
Rat

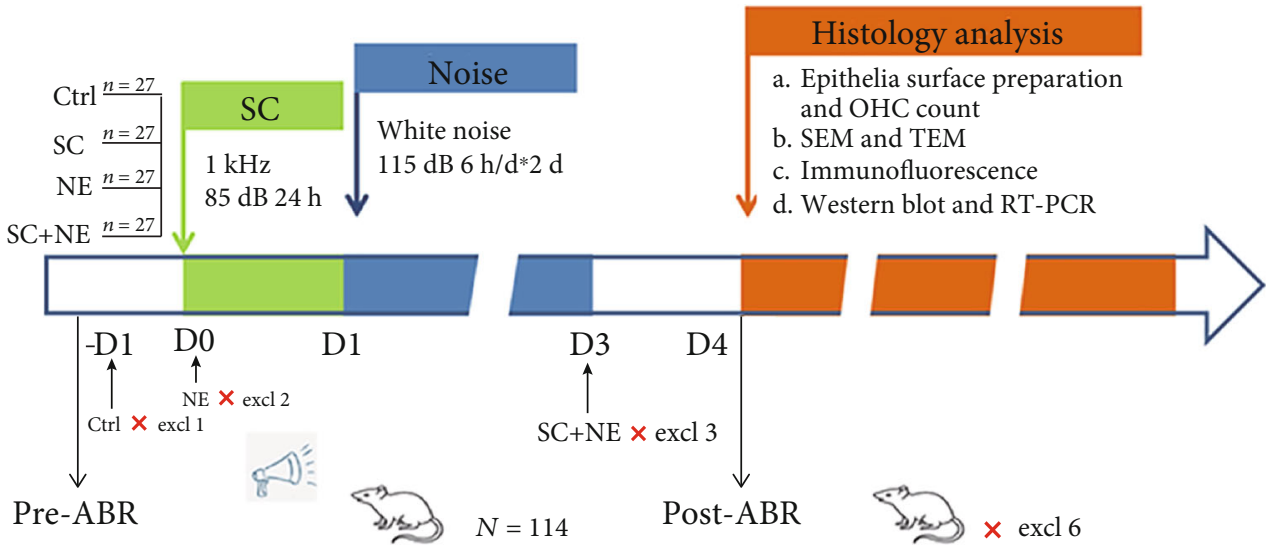

(a)

Neonatal rat P1-3d

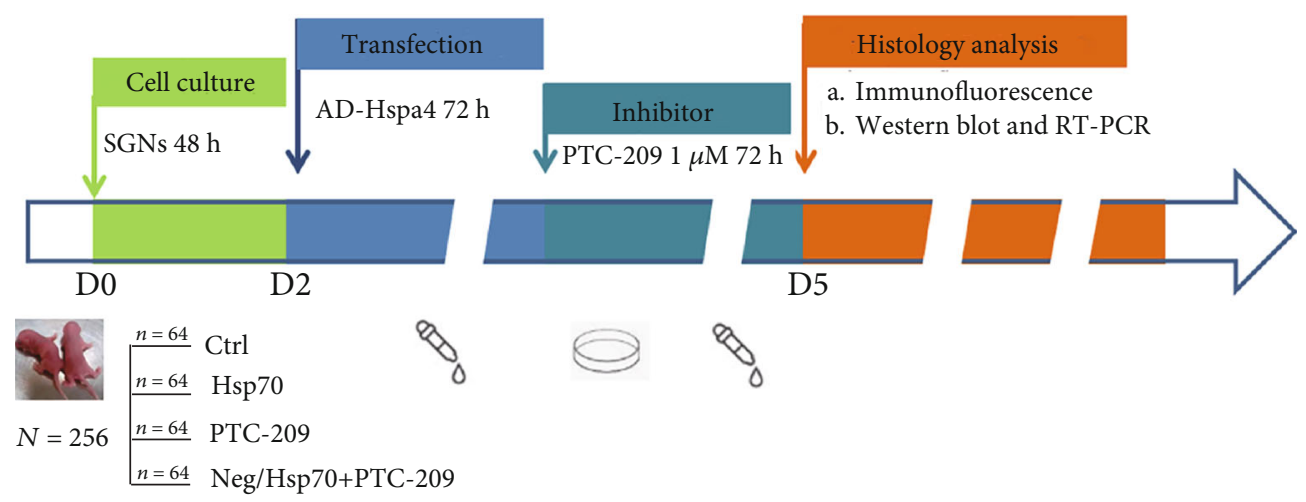

(b)

Figure 1: Chronogram of the experiment in vivo (a) and in vitro (b). (a) 114 rats were available in the experiment in vivo, but there were six animals that were excluded based on the exclusion criteria (tympanitis) or died during experiments. Rats were exposed to a pure tone of $1 \mathrm{kHz}$ at $85 \mathrm{~dB}$ SPL (SC) for 24 hours; then, some of them and others were exposed to white noise at $115 \mathrm{~dB}$ SPL (NE) for 6 hours per day over two consecutive days with anesthesia; they were allowed to rest for 3 hours between SC and NE with anesthesia. Hearing was evaluated with auditory brainstem responses (ABRs) before 1 day prior to exposure and 1 day after exposure. At the end of the study in vivo, cochleae were dissected and processed for histology analysis. (b) The spiral ganglion neuron cells (SGNs) from 256 neonatal rats were harvested and then cultured on culture dishes for $48 \mathrm{~h}$; next, AD-Hspa4 (Hsp70-overexpressing adenovirus) and $1 \mu \mathrm{M}$ PTC-209 (a small-molecule inhibitor of Bmil) were, respectively, added to the appropriate cells and incubated for 72 hours at $37^{\circ} \mathrm{C}$. At the end of the study in vitro, SGNs were processed for histology analysis.

Deeply anesthetized animals from each group were perfused transcardially with $0.9 \%$ saline followed by a fixative solution of $2.5 \%$ glutaraldehyde and $4 \%$ paraformaldehyde in 0.1 M PBS (pH 7.4) immediately after the ABR measurements. The cochleae were removed immediately, maintained in the same solution at $4^{\circ} \mathrm{C}$ for at least 24 hours, and decalcified with $10 \%$ EDTA at $23^{\circ} \mathrm{C}$ for two weeks. Following washing with 0.1 M PBS, the cochleae were divided into small blocks of approximately $1 \mathrm{~mm}^{3}$ in size under a dissecting microscope (Olympus, Japan), then fixed with 1\% osmic acid for 2 hours, gradually dehydrated in gradient acetone, and embedded in Epon 812 for polymerization. Next, the sample blocks were sectioned at a $10 \mu \mathrm{m}$ thickness and stained with Evans blue to visualize the spiral ganglion. Following visualization, ultrathin $70 \mathrm{~nm}$ thick sections were prepared and stained with uranyl acetate and lead citrate following a conventional protocol.
Finally, the ultrathin sections were observed under TEM (JEM, Japan) to reveal the ultrastructure of SGNs in the cochlea. The experimenter of electron microscopy was unaware of the animal's group during the experimentation of observation.

2.4. Western Blot. Twelve deeply anesthetized rats from each group were sacrificed immediately after the last ABR measurement by decapitation on ice, and the modiolus tissues (or SGN cells in vitro) from the same group were harvested, pooled, and stored at $-80^{\circ} \mathrm{C}$ until use. The pooled tissues were lysed in sample buffer containing $1 x$ Tris-EDTA, $\mathrm{NaCl}$ (100 $\mathrm{mM}), 1 \%$ Triton $\mathrm{X}-100$, and $1 \mathrm{x}$ protease inhibitors. Following mechanical lysis and protein extraction, protein concentration was determined by using a BCA assay kit (Millipore, catalog number BCA1-1KT). Equal amounts 


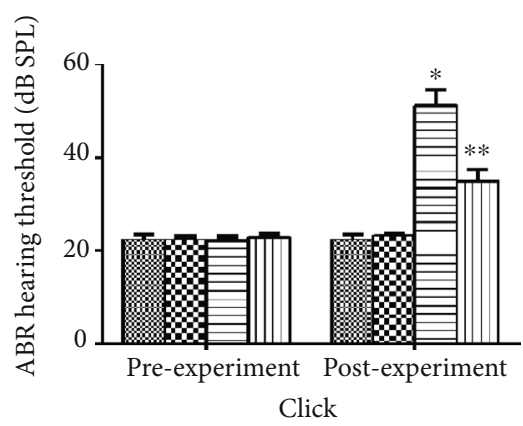

$89 \operatorname{Ctrl}(n=6)$

SC $(n=6)$

$\boxminus \mathrm{NE}(n=6)$

WI SC+NE $(n=6)$

(a)

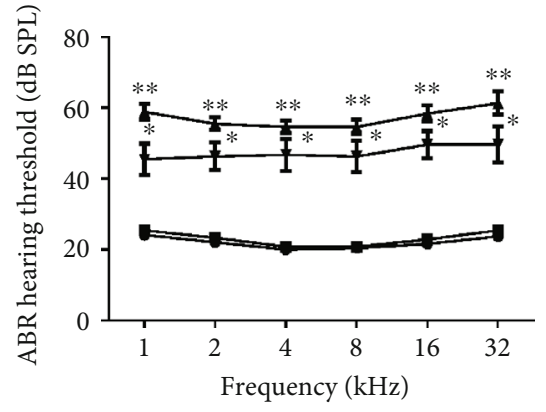

$\longrightarrow \operatorname{Ctrl}(n=6)$

$\Rightarrow$ SC $(n=6)$

$\neg \mathrm{NE}(n=6)$

$\neg \mathrm{SC}+\mathrm{NE}(n=6)$

(b)

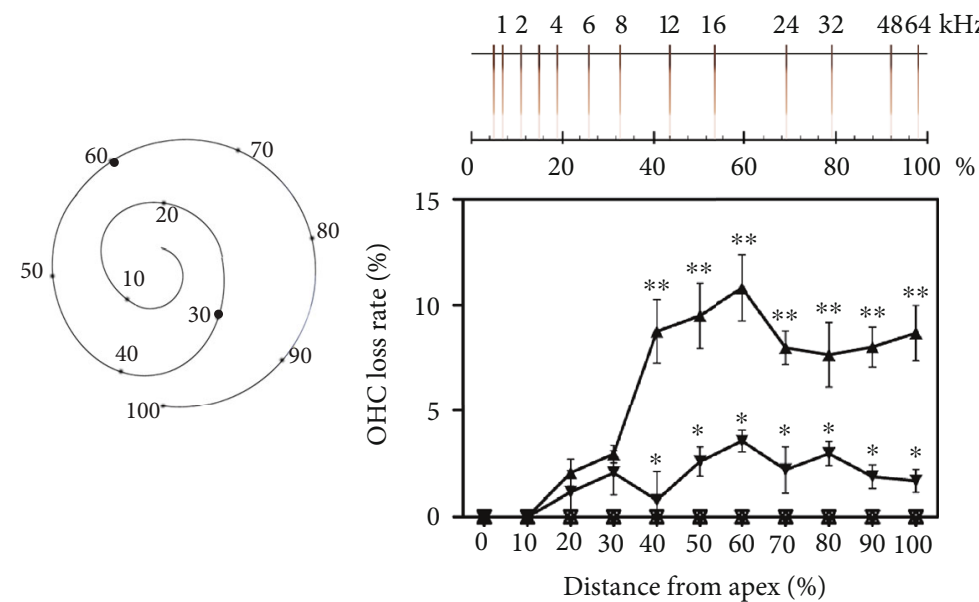

$\begin{array}{llll}\triangle & \text { Ctrl } & \boldsymbol{\nabla} & \text { NE } \\ \nabla & \text { SC } & \boldsymbol{\nabla} & \text { SC+NE }\end{array}$

(c)

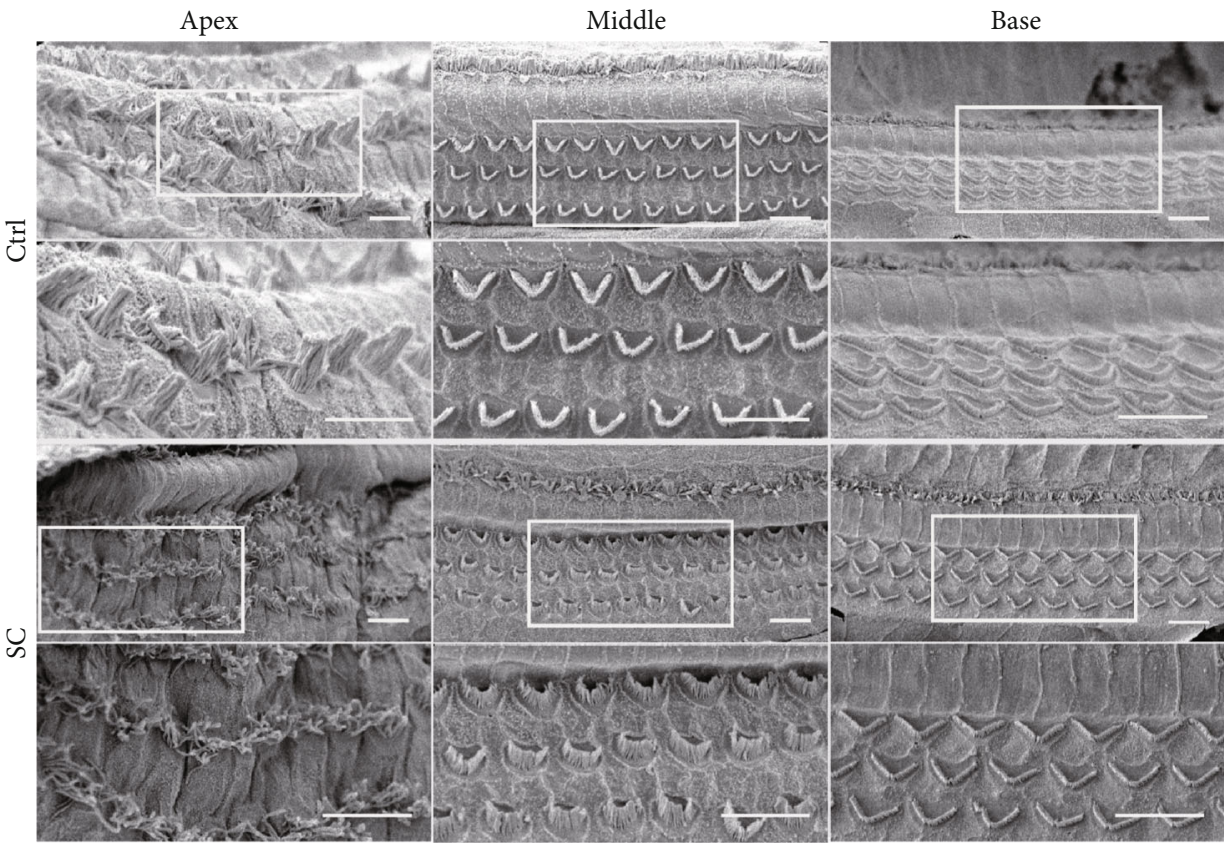

(d)

Figure 2: Continued. 


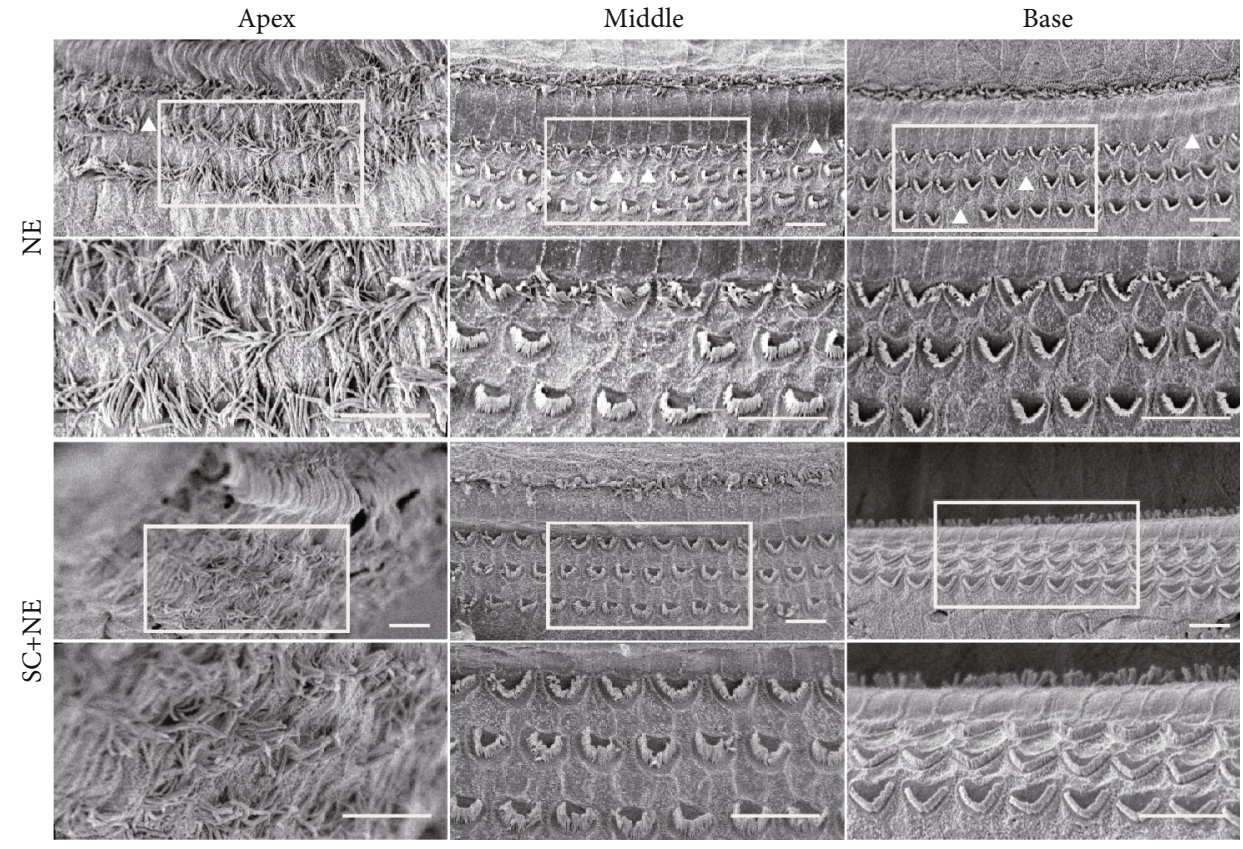

(e)

FIGURE 2: ABR click and tone and basilar membrane and OHC loss. (a) Hearing thresholds of click sound from four groups before and after noise exposure (NE vs. Ctrl: ${ }^{*} P<0.01$, SC+NE vs. NE: ${ }^{* *} P<0.01$; mean $\pm \mathrm{SEM}, n=6$ animals/group). (b) Hearing thresholds of pure tone from four groups after noise exposure (NE vs. Ctrl: ${ }^{*} P<0.05$, SC+NE vs. NE: ${ }^{* *} P<0.01$; mean \pm SEM, $n=6$ animals/group). Ctrl: control group; SC: sound conditioning group which was exposed to a pure tone of $1 \mathrm{kHz}$ at $85 \mathrm{~dB}$ SPL for 24 hours; NE: noise exposure group which was exposed to white noise at $115 \mathrm{~dB}$ SPL for 6 hours per day over 2 consecutive days; SC+NE: sound conditioning and noise exposure group which was exposed to a pure tone of $1 \mathrm{kHz}$ at $85 \mathrm{~dB}$ SPL for 24 hours, and then 3 hours later, followed by white noise at $115 \mathrm{~dB}$ SPL for 6 hours per day over 2 consecutive days. Scanning electron micrographs for OHCs in different turns of basilar membrane from the Ctrl and SC groups (d) as well as the NE and SC+NE groups (e). The lower row pictures are the upper row ones at high magnification. White frames show the enlarged areas; triangular arrows indicate OHC loss. Scale bars represent $10 \mu \mathrm{m}$. Percentage of $\mathrm{OHC}$ loss in different turns from four groups presented in (c) was analyzed with Tukey's multiple comparisons test (Base $\mathrm{NE}_{\mathrm{vs} .} \mathrm{SC}+\mathrm{NE}:{ }^{*} P$ $<0.05$, Middle $\mathrm{NE}_{\mathrm{vs} . \mathrm{SC}+\mathrm{NE}}{ }^{* *} \mathrm{P}<0.05$; mean $\pm \mathrm{SD}, n=6$ pictures from 3 animals/group). Scale is showing frequency and percent distance from the apex for rat cochlea according to Muller (1991). The apex turn of the basilar membrane was considered as the low-frequency area of 0 to 30 percent distance from the apex for the cochlea; the middle turn was the middle-frequency area of 30 to 60 percent distance from the apex, and the base turn was the high-frequency area of 60 to 100 percent distance from the apex.

(approximately $100 \mathrm{ng} /$ lane) of protein were loaded on $12 \%$ sodium dodecyl sulfate-polyacrylamide gels (SDS-PAGE) (Bio-Rad, catalog number 1610173) (120 V for $80 \mathrm{~min}$ at room temperature), electrophoresed, and transferred to PVDF membranes (Sigma-Aldrich, catalog number GE10600122) by electroblotting $\left(200 \mathrm{~mA}\right.$ for $40 \mathrm{~min}$ at $\left.4^{\circ} \mathrm{C}\right)$ in $1 \mathrm{x}$ transfer buffer (Sigma-Aldrich, catalog number PCG3011). After being blocked in 5\% nonfat dry milk and $0.1 \%$ Tween-20 in PBS ( $\mathrm{pH} 7.4,0.01 \mathrm{M})$ (Sigma-Aldrich, catalog number 9005-64-5) for 1 hour at $25^{\circ} \mathrm{C}$, the membranes were incubated overnight at $4^{\circ} \mathrm{C}$ with different primary antibodies (rabbit anti-FoxO1 polyclonal antibody, 1:1000, Cell Signal, catalog number 2880; rabbit anti-Hsp70 polyclonal antibody, $1: 1000$, Abcam, catalog number ab79852; rabbit anti-Bmil polyclonal antibody, 1:1000, Abcam, catalog number ab38295; rabbit anti-SOD1 polyclonal antibody, 1:1000, Proteintech, catalog number 10269-1-AP; or rabbit anti-SOD2 polyclonal antibody, 1:1000, GeneTex, catalog number GTX116093). The membranes were then incubated with an HRP-conjugated secondary antibody (goat anti-rabbit antibody, Bioworld, catalog number BS13278) for 1 hour at room temperature. Equal protein loading was confirmed by stripping the blots and reprobing them with a polyclonal rabbit anti- $\beta$-actin antibody (1:1000, GeneTex, catalog number GTX109639) followed by incubation with the same HRP-conjugated secondary antibody. The protein bands were detected by using a chemiluminescence detection technique (FluorChem, Alpha Innotech, USA), and the gray density of the detected bands was analyzed with NIH ImageJ software. The experimenter of western blot was unaware of the animal's group during the experimentation.

2.5. Reverse Transcription and Quantitative Real-Time PCR (Quantitative RT-PCR). This assay was used to detect mRNA expression levels of Hsp70, Bmi1, SOD1, and SOD2 in the rat spiral ganglion cells from the four groups. Total RNA was isolated by TRIzol reagent (Life Technologies Corporation, catalog number 15596026) according to the manufacturer's instructions. Reverse transcription was performed with a First Strand cDNA Synthesis Kit (GeneCopoeia, catalog number AORT-0020) according to the manufacturer's instructions. Quantitative RT-PCR was performed with an All-in-One qPCR Mix (GeneCopoeia, catalog number QP001). Quantitative RT-PCR primers for Hsp70 (RQP051424), Bmil 
TAble 1: Primers used for quantitative RT-PCR.

\begin{tabular}{lccccc}
\hline Gene & Lot No. & Primer ID & Amplicon size & GenBank accession number & Annealing temperature \\
\hline Hsp70 & RQP051424 & Rn-QRP-10166 & 133 & NM_031971.2 & $60^{\circ} \mathrm{C}$ \\
Bmi1 & RQP083017 & Rn-QRP-10984 & 119 & NM_001107368.2 & $60^{\circ} \mathrm{C}$ \\
SOD1 & RQP049577 & Rn-QRP-10681 & 128 & NM_017050.1 & $60^{\circ} \mathrm{C}$ \\
SOD2 & RQP049578 & Rn-QRP-10168 & 91 & NM_017051.2 & $60^{\circ} \mathrm{C}$ \\
$\beta$-Actin & RQP051050 & Rn-QRP-10046 & 98 & NM_031144.2 & $60^{\circ} \mathrm{C}$ \\
\hline
\end{tabular}

(RQP083017), SOD1 (RQP049577), SOD2 (RQP049578), and $\beta$-actin (RQP051050) were purchased from GeneCopoeia (GeneCopoeia, China). The forward and reverse primers of each PCR set, the sizes of PCR products, GenBank accession numbers, primer IDs, and annealing temperatures are presented in Table 1. RT-PCR was performed for forty cycles with the following parameters: $10 \mathrm{~min}$ at $95^{\circ} \mathrm{C}$ for predenaturation, and in each cycle, $10 \mathrm{~s}$ at $95^{\circ} \mathrm{C}$ for denaturing, $30 \mathrm{~s}$ at $60^{\circ} \mathrm{C}$ for annealing, and $15 \mathrm{~s}$ at $72^{\circ} \mathrm{C}$ for extending (Bio-Rad, USA). All quantitative RT-PCR analyses were conducted with the CFX Manager 3.0 (Bio-Rad, USA). Expression levels of Hsp70 and other genes were normalized to that of $\beta$-actin by the delta Ct value. The experimenter of PCR was unaware of the animal's group during the experimentation.

2.6. Immunofluorescence. Rats from each group were perfused transcardially with freshly prepared $4 \%$ paraformaldehyde in 0.1 M PBS ( $\mathrm{pH} 7.4$ ) under deep anesthesia with pentobarbital sodium $(60 \mathrm{mg} / \mathrm{kg})$ after ABR measurements. The cochleae were postfixed with the same fixative at $4^{\circ} \mathrm{C}$ for at least 24 hours, decalcified with $10 \%$ EDTA at $23^{\circ} \mathrm{C}$ for 1 week, dehydrated in 30\% sucrose for 24 hours, embedded in OCT glue (SAKURA, catalog number 4583), and sectioned at a $10 \mu \mathrm{m}$ thickness in the midmodiolus plane on a cryostat (Leica, Germany). The sections were soaked with $0.3 \%$ hydrogen peroxide in methanol for $10 \mathrm{~min}$ to inactivate endogenous peroxidase and blocked with goat serum (Abcam, catalog number ab7481) at $37^{\circ} \mathrm{C}$ for $30 \mathrm{~min}$. Then, they were incubated with rabbit anti-Hsp70 polyclonal antibody $(1: 100$, Abcam), rabbit anti-Bmil polyclonal antibody (1:100, Abcam), rabbit anti-FoxO1 monoclonal antibody (1:100, Cell Signal), rabbit anti-SOD1 polyclonal antibody (1:100, Proteintech) or rabbit anti-SOD2 polyclonal antibody (1:100, GeneTex), MitoSOX ${ }^{\mathrm{TM}}$ Red mitochondrial superoxide indicator for live-cell imaging (5uM, Invitrogen, catalog number M36008), and mouse anti-tubulin monoclonal antibody $\left(1: 100\right.$, Abcam, catalog number ab7751) at $4^{\circ} \mathrm{C}$ overnight. Primary antibodies were omitted from the negative controls for these antibodies. Then, sections were incubated with Alexa Fluor 488 Donkey anti-Mouse IgG Highly Cross-Adsorbed Secondary Antibody (1:200, Life Technologies, catalog number A-21202) or Alexa Fluor 647 Donkey anti-Mouse IgG Highly Cross-Adsorbed Secondary Antibody (1:200, Life Technologies, catalog number A-31571) and Alexa Fluor 594 goat anti-rabbit IgG Highly Cross-Adsorbed Secondary Antibody $(1: 200$, Life Technologies, catalog number A-11012) at $37^{\circ} \mathrm{C}$ for $30 \mathrm{~min}$, followed by staining with DAPI or Hoechst $33258(1: 1000$, Boster, catalog number
AR1176 or catalog number AR1169) at $37^{\circ} \mathrm{C}$ for $10 \mathrm{~min}$. Three washes in $0.01 \mathrm{M}$ PBS were carried out in the intervals of each step above. Next, the sections were mounted on collagencoated glass slides and visualized under a fluorescence microscope (Olympus, Japan), while the quantity of target protein expression was analyzed by fluorescence intensity. The experimenter of the fluorescence microscope was unaware of the animal's group during the experimentation of observation.

2.7. Cell Culture and Transfection. In this study, 256 healthy neonatal Sprague-Dawley rats (P1-3d) were used for cell culture. Before the experiment starts, cell culture preparation including sterilization and disinfection of cell culture room and operations area and disinfection of work clothes, aseptic mask, sterile gloves, and operating instruments should be strictly implemented, and animals should also be disinfected in time before they are killed quickly. The disinfected animals were sacrificed immediately by decapitation on ice with anesthesia in the disinfected operation room. Then, the modiolus tissues (SGNs) from the killed animals' cochleae were dissected, harvested, and pooled on ice under the stereomicroscope (Leica, Germany) in a super clean bench. After treatment with $0.125 \%$ collagenase IV (Thermo Fisher Scientific, catalog number 17104019 ) and $0.25 \%$ trypsinase (Thermo Fisher Scientific, catalog number 25200-056), the spiral ganglion neuron cells (SGNs) from 20 newborn rats per time were cultured on culture dishes in SGN culture medium Dulbecco's modified Eagle's medium with 2\% B27 (Thermo Fisher Scientific, catalog number 17504-044), $10 \mu \mathrm{g} / \mathrm{ml}$ brain-derived neurotrophic factor (BDNF, PeproTech, catalog number $450-02)$, and $1 \%$ penicillinstreptomycin solution (100,000 U/l, HyClone, catalog number SV30010) $[26,27]$. Then, the SGNs were cultured at $37^{\circ} \mathrm{C}$ in a humidified incubator (Thermo Scientific, USA) with $5 \% \mathrm{CO}_{2}$ for 5 days, and the medium was refreshed every 2-3 days. Following a 48-hour incubation, the culture dish of SGNs was divided into four dishes for different interventions, to which $1 \mu \mathrm{M}$ PTC-209 (a specific inhibitor of Bmil with an IC50 value of $0.5 \mu \mathrm{M}$, Selleckchem, CAS No. 315704-66-6, catalog number S7372), AD-Hspa4 $(2 E+10 \mathrm{PFU} / \mathrm{ml}$, 17999-1, GeneChem, China), AD-CON177 $(3 E+10$ $\mathrm{PFU} / \mathrm{ml}$, CMV-MCS-3FLAG-SV40-EGFP, GeneChem, China), and enhanced infection solution (GeneChem, China) were added to the appropriate cells and incubated for 72 hours at $37^{\circ} \mathrm{C}$. Finally, we observed and disposed of the SGNs from the different procedures [28] (Figure 1(b)). The experimenter of cell culture was unaware of the animal's group during the experimentation of observation. 
2.8. Statistical Analysis. Experiments were replicated a minimum of three times. The group size $(n)$ in vivo was determined by the variability of measurements and the magnitude of the differences between groups. Based on our previous as well as current preliminary studies and sample size calculation (Type I error probability $\alpha$, type II error probability $\beta$, permissible error $\delta$, and standard deviation $S$ were used to estimate effect size, when the formula of the sample size required for comparison of means of two samples was performed $\left(n_{1}=n_{2}=2\left[\sigma\left(u_{\alpha}+u_{\beta}\right) / u\right]^{2}+u_{\alpha}{ }^{2} / 4\right)$.), we determined that six animals per group provide sufficient statistical power (While double-tailed $\alpha$ was 0.05 and single-tailed $\beta$ was 0.1 in our study, $u_{\alpha / 2}$ was 1.96 and $u_{\beta}$ was 1.282. According to our protocol, standard deviation $S$ equaled to $\sigma$ was 2.4 and permissible error $\delta$ was 5. Finally, the number of animals per group was 5.80 based on the formula of sample size calculation.). Data are presented as means $\pm \mathrm{SD}$ (standard deviations) or means \pm SEM (standard error of mean). The assessment (Q-Q plot) of the normality of data was carried out by SPSS version 17.0 software package (SPSS Inc., Chicago, Ill.). The test (linear regression model) for outliers was conducted on the data by SPSS Data Validation, and no data point was excluded. Two-way analysis of variance was used for comparisons among the different groups that were impacted by two factors, and comparisons with more than two groups were performed with one-way analysis of variance (ANOVA). Further tests included the Student-Newman-Keuls- (SNK-) $q$ test for post hoc comparisons or Tukey's multiple comparisons test using GraphPad Prism 5. Statistical analysis of the comparisons between the two groups was accomplished by Student's $t$ -test. All tests were two-tailed, and differences were considered to be statistically significant at $P<0.05$. The experimenters were unaware of the animal's group during experimentation and statistical analysis. Furthermore, the analysis or experimental group assignment was performed by a different person than the experimenter, while all rats were randomly divided into the groups by a simple randomization protocol described in the following. Thus, blinding was achieved in our study.

2.9. Compliance with Ethical Standards. All procedures concerning animals in this study were approved by the Institutional Animal Care and Use Committee of the Fourth Military Medical University (Permit number, SYXK 2008005) and were in compliance with the Guide for the Care and Use of Laboratory Animals.

\section{Results}

3.1. SC Protection against Hearing Loss Caused by Acute Noise Exposure. ABR click and tone burst profiles were successfully obtained from rats in each group one day before and after exposure. One-way ANOVA can detect significant changes in hearing function (Figure 2(a)). There was a significant group difference found in postthreshold $(F=38.96, P$ $<0.0001)$ among the four groups. In particular, SNK- $q$ tests revealed that there was a significant difference found in the postthreshold between the NE and Ctrl groups $(q=13.37$, * $P<0.01)$ and between the NE and SC+NE groups $(q=7.56$, $\left.{ }^{* *} P<0.01\right)$. However, no significant difference was found between the SC and Ctrl groups $(q=0.39, P>0.01)$.

The ABR hearing threshold for pure tone frequencies was calculated via two-way ANOVA to detect significant changes in the hearing function (Figure 2(b)). Notably, there was a significant group difference $(F=302.20, P<0.0001)$ found among three of the groups (the NE, SC+NE, and Ctrl groups). SNK- $q$ tests also showed that there was a significant difference found in the postthreshold between the NE and Ctrl groups $\left(q=76.28,{ }^{* *} P<0.001\right)$ and between the $\mathrm{NE}$ and SC+NE groups $\left(q=15.88,{ }^{*} P<0.001\right)$. However, no significant difference was found between the SC and Ctrl groups $(q=8.944, P>0.001)$. All of these data supported our concept that SC protected against AAT but did not result in hearing impairment on fully mature rats.

3.2. Effects of SC and Acute Noise Exposure on Hair Cell Structure. Scanning electron microscopy (SEM) revealed different effects of SC and acute noise exposure on the basilar membrane structure. The results showed that the stereocilia were well organized in "V" or "W" shapes in the Ctrl group. Furthermore, SC resulted in the functional reconstruction of OHCs which included shortening and thickening of the cilia except for misalignment and loosening of the outer hairs in the apex turn of the basilar membrane, while acute noise exposure leads to $\mathrm{OHC}$ loss, lodging, fusion, and disappearance of cilia (Figures 2(d) and 2(e)).

Moreover, we analyzed the proportion of OHC loss among the different groups from the base to the apex turns (Figure 2(c)). Tukey's multiple comparisons test revealed that there was a noticeable hair cell loss in both the base and middle turns of the basilar membrane in the NE group compared with the Ctrl group $(F=235.5$ (column factor: treatment), $P<0.0001 ; q_{B}=23.92,{ }^{*} P<0.05 ; q_{M}=28.68,{ }^{*}$ $P<0.05)$, while no significant differences were found between these two groups in the apex turn of the basilar membrane $\left(q_{A}=3.70, P>0.05\right)$. This finding suggested that acute noise exposure could clearly result in OHC loss in both the base and middle turns but not the apex turn of the basilar membrane. Furthermore, compared with the NE group, less hair cell loss in both the base and middle turns of the basilar membrane was found in the SC+NE group along with shortening and thickening of the cilia, while misalignment and loosening of the outer hairs in the apex turn of the basilar membrane were not improved $\left(q_{B^{\prime}}=17.40,{ }^{* *} P<0.05 ; q_{M^{\prime}}\right.$ $\left.=21.82,{ }^{* *} P<0.05\right)$. However, this group exhibited no obvious hair cell loss in the apex turn of the basilar membrane with the same reconstruction of OHCs $\left(q_{A^{\prime}}=\right.$ $1.31, P>0.05)$. This result suggested that SC could protect against $\mathrm{OHC}$ loss caused by NE in both the base and middle turns of the basilar membrane on fully mature rats.

\subsection{Ultrastructural Changes in SGNs Caused by SC and Acute} Noise Exposure. Transmission electron microscopy (TEM) revealed ultrastructural changes in SGNs caused by SC and acute noise exposure on fully mature rats. SC increased the number of mitochondria in the SGNs, enhanced the electron density of the mitochondria in SGNs, and narrowed the interspace of the mitochondrial matrix. Conversely, NE 


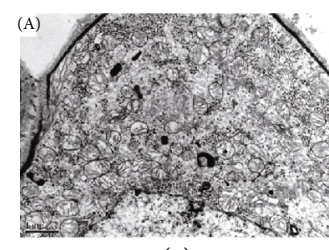

(a)

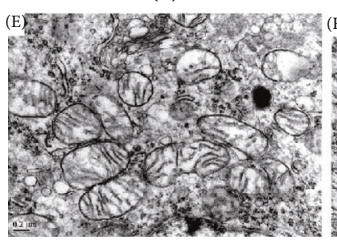

$\left(a^{\prime}\right)$

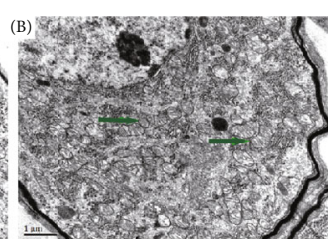

(b)

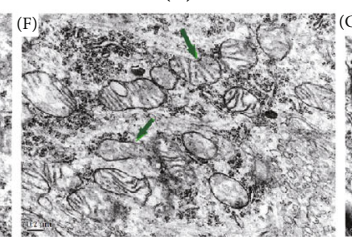

$\left(b^{\prime}\right)$

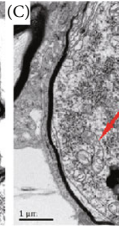

(c)

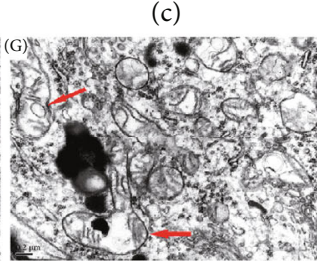

$\left(c^{\prime}\right)$

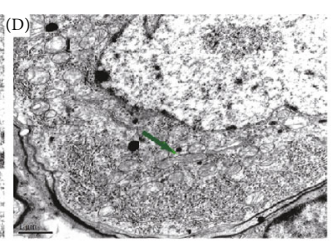

(d)

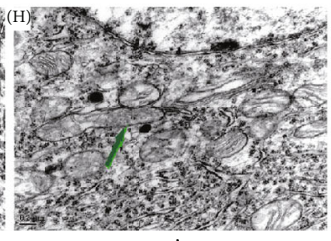

$\left(d^{\prime}\right)$

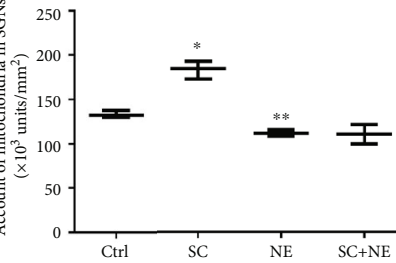

(e)

FIGURE 3: Ultrastructural changes of spiral ganglion neuron. Electron transmission micrographs for SGNs in the control group (a/a'), sound conditioning group $\left(\mathrm{b} / \mathrm{b}^{\prime}\right)$, noise exposure group $\left(\mathrm{c} / \mathrm{c}^{\prime}\right)$, and sound conditioning and noise exposure group $\left(\mathrm{d} / \mathrm{d}^{\prime}\right)$. Green arrows showed that sound conditioning could enhance the electron density in the mitochondrion of spiral ganglion neuron cells and narrow the interspace of the mitochondrion. Red arrows indicated that noise exposure could result in the destruction of the mitochondrion in SGNs, the lower electron density, larger interspace, and the formation of the vacuole in the mitochondrion. Scale bars represent $1 \mu \mathrm{m}$ and $0.2 \mu \mathrm{m}$. The account of a mitochondrion in spiral ganglion neuron cell of SD rat presented in (e) was analyzed with one-way ANOVA $(F=55.17 ; P<0.0001)$, followed by Newman-Keuls' post hoc test $\left(\times 10^{3}\right.$ units $/ \mathrm{mm}^{2}, n=6$ pictures from 3 animals/group, mean \pm SD; Ctrl vs. NE: ${ }^{* *} P<0.05$, SC vs. Ctrl: $\left.{ }^{*} P<0.05\right)$.

resulted in the shrinkage of SGNs, decreased the numbers of mitochondria in the SGNs, lowered electron density, and enlarged the interspace between the mitochondrial matrix and the formation of vacuoles in the mitochondria (Figure 3).

We calculated the number of mitochondria in the SGNs of fully mature rats and analyzed their density by the SNK$q$ test. It showed that the densities of mitochondria were significantly different between the $\mathrm{NE}$ and Ctrl groups $\left(q=4.78,{ }^{* *} P<0.05\right)$ and between the SC and Ctrl groups $\left(q=10.86,{ }^{*} P<0.05\right)$ (Figure 3(e)). This result indicated that $\mathrm{SC}$ increased the quantity of mitochondria while NE reduced it. But there was no difference between the account of mitochondria in the $\mathrm{SC}+\mathrm{NE}$ and $\mathrm{NE}$ groups shown in Figure 3(e) $(q=0.23, P>0.05)$. It means that NE nullified the protection of mitochondria by SC and SC could largely enhance the density of mitochondria to protect against NEinduced trauma instead of increasing the quantity of mitochondria.

3.4. Effects of SC and Acute Noise Exposure on Hsp70, Bmil, SOD1, SOD2, and FoxO1 Expression Levels in the Rat Spiral Ganglion Cells. The IF results revealed that NE lowered Hsp70, Bmil, FoxO1, SOD1, and SOD2 fluorescence levels in SGNs of a fully mature rat, while SC increased Hsp70, Bmil, FoxO1, SOD1, and SOD2 fluorescence levels in SGNs (Supplementary Table 1). The WB results of decline at the protein level of Hsp70, Bmi1, SOD1, and SOD2 expressions were found in the NE group, while increased expression of Hsp70, Bmi1, SOD1, and SOD2 expression was found in the SC group (Figures 4(a), 5, and 6 and Supplementary Table 2). In addition, the IF results also revealed that NE lowered Hsp70, Bmi1, SOD1, and SOD2 fluorescence levels in both hair cells and cochlear lateral wall of a fully mature rat, while SC increased Hsp70, Bmi1, SOD1, and SOD2 fluorescence levels in these two regions (Supplementary Figure 3 and 4 and Supplementary Table 1).
Furthermore, the results indicated that SC reversed the downregulation of Hsp70, Bmi1, FoxO1, SOD1, and SOD2 expression caused by NE in SGNs of a fully mature rat. Moreover, RT-PCR exhibited that Hsp70, Bmi1, SOD1, and SOD2 mRNA expression was increased in the SGNs of the SC group compared with that of the Ctrl group $\left({ }^{*} P<0.05 ; n=6\right)$ (Figure 4(b)).

3.5. Overexpression of Hsp70 Significantly Enhanced Bmil, SOD1, SOD2, and FoxO1 Expression in Rat SGNs and Decreased ROS Accumulation In Vitro. Analysis of the neuronal cells on neonatal cultures expressing a green fluorescent protein (GFP) coded in the same vector as the Hsp70overexpressing vector was coupled to the GFP sequence, which indicated that $66.7 \% \pm 7.8 \%(n=4$ independent cell culture preparations) of the neuronal cells present in the cultures were transfected with Hsp70. After the transfection of SGNs with Hsp70-overexpressing adenovirus in vitro, IF results (Figures $7(\mathrm{a})-7(\mathrm{~d}) / 7\left(\mathrm{a}^{\prime}\right)-7\left(\mathrm{~d}^{\prime}\right)$ and Supplementary Table 1) revealed that ratios of Bmi1, SOD1, SOD2, and FoxO1 fluorescence levels were all increased in the SGNs of neonatal culture compared with the negative control.

Following transfection of the SGNs with Hsp70 overexpressing the adenovirus on neonatal cultures in vitro, WB results (Figure $7(\mathrm{e}) / 7\left(\mathrm{e}^{\prime}\right)$ and Supplementary Table 2) also revealed that similar results were found at the protein level of Hsp70, Bmi1, FoxO1, SOD1, and SOD2 expression. There were clearly significant differences between the Hsp70overexpressing adenovirus and negative control groups $\left(F=3400.31,{ }^{* *} P<0.05\right)$. Moreover, RT-PCR revealed that Hsp70, SOD1, and SOD2 mRNA expression increases in the SGNs of neonatal culture except for Bmil mRNA expression (Figure 8(f) and Supplementary Figure 2).

In addition, there was a significant difference between the Hsp70-overexpressing adenovirus and negative control groups in ROS accumulation labeled by MitoSOX Red (a 

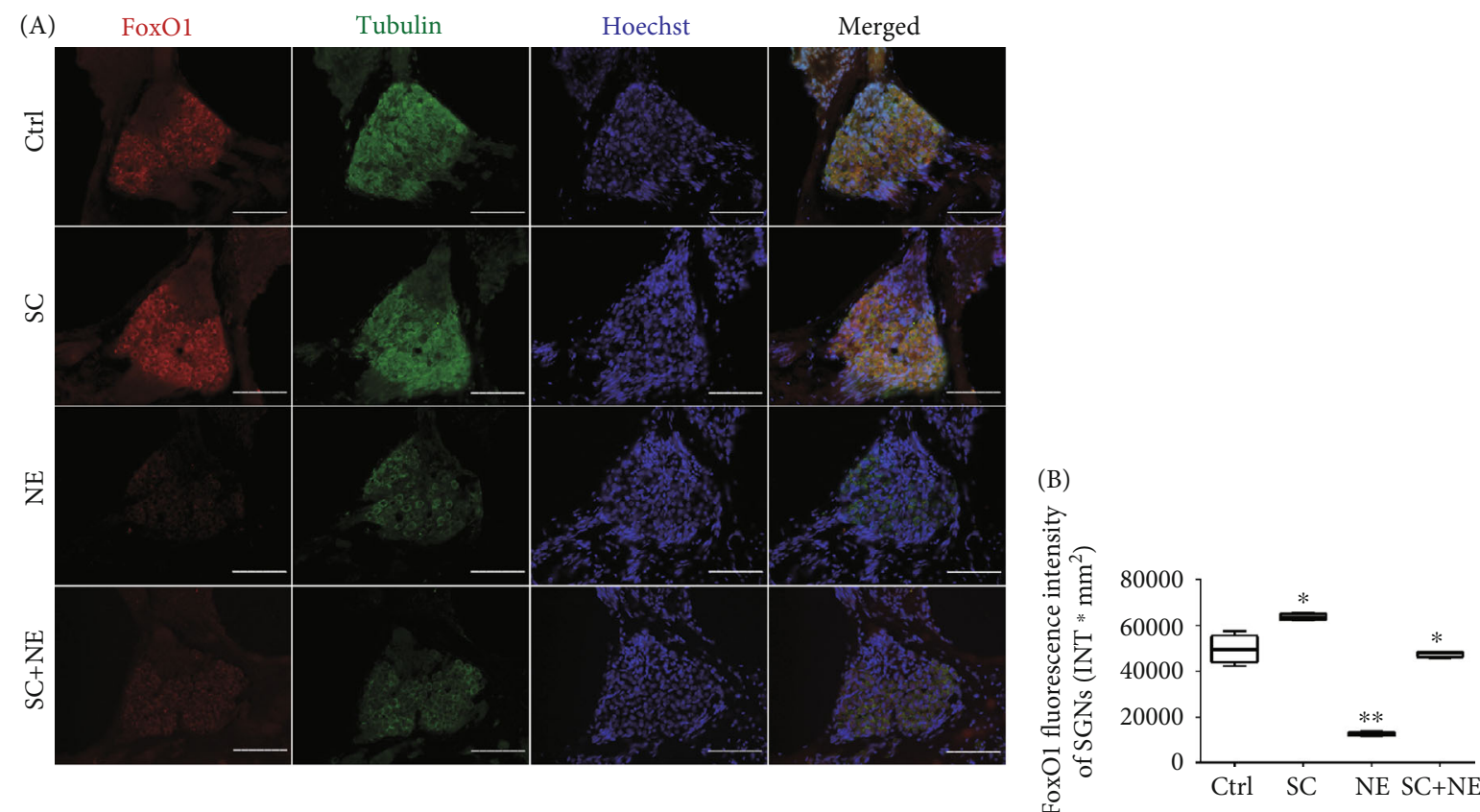

(a)

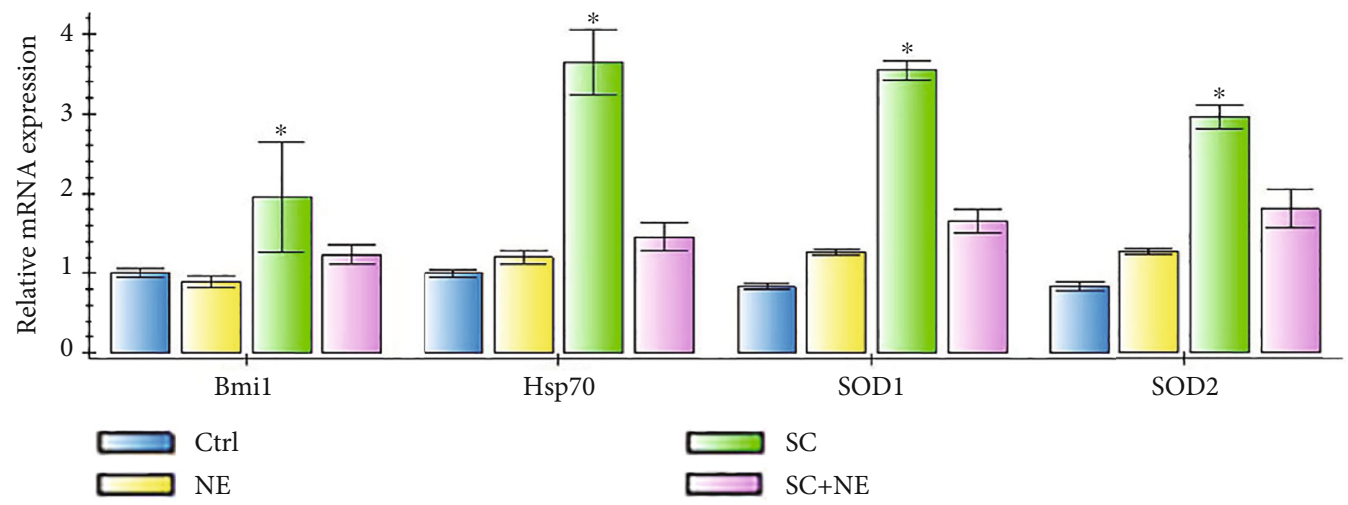

(b)

FIGURE 4: FoxO1 protein and Hsp70, Bmil, SOD1, and SOD2 mRNA expressions of SGNs after sound conditioning and acute noise exposure. Distributions and expressions of FoxO1 (a) protein were detected with the immune-fluorescence assay in the Ctrl and SC groups as well as the NE and SC+NE groups (A), while the quantity of these protein expressions was analyzed by fluorescence intensity (B). FoxO1 (red) and tubulin (green) as SGN marker were, respectively, labeled with fluorescent secondary antibody, and nuclei (blue) were labeled with Hoechst. Scale bars represent $100 \mu \mathrm{m}$ (a). Statistical analysis of the results presented in (B) was performed with one-way ANOVA (FoxO1: $F=155.3, P<0.0001$ ), followed by Newman-Keuls' post hoc test $\left({ }^{*} P<0.05,{ }^{* *} P<0.05 ; n=6\right.$ pictures from 3 animals/group). Furthermore, values are means \pm SD. Relative mRNA expressions of Bmil, Hsp70, SOD1, and SOD2 were determined by quantitative RT-PCR (b). $\beta$-Actin RNA level was used as an endogenous control. Values are means \pm SD $\left(n=6\right.$ animals/group). ${ }^{*} P<0.05$ vs. control group.

mitochondrial superoxide indicator for live cells), and the Hsp70-overexpressing adenovirus group $\left(q=31.64,{ }^{* *} P<\right.$ 0.05 ) showed an apparent decrease in ROS accumulation of neonatal culture in vitro (Figure $8(\mathrm{a}) / 8(\mathrm{c}) / 8(\mathrm{e})$ ).

3.6. PTC-209 Decreased the Expression of Bmi1, SOD1, and SOD2 in Rat SGNs and Significantly Increased ROS Accumulation in Rat SGNs In Vitro. Following treatment with PTC-209 on neonatal cultures in vitro, IF results (Figures $9(\mathrm{a}) / 9\left(\mathrm{a}^{\prime}\right), 9(\mathrm{c}) / 9\left(\mathrm{c}^{\prime}\right)$, and $9(\mathrm{~d}) / 9\left(\mathrm{~d}^{\prime}\right)$ and Supplementary Table 1) revealed that Bmil, SOD1, and SOD2 fluorescence levels were all decreased in SGNs compared with those in the control group. There were clearly significant differences between the PTC-209 and control groups except for the Hsp70 fluorescence levels $(t=0.8032, P>0.05)$ (Supplementary Figure 1). WB results (Figure $9(\mathrm{e}) / 9\left(\mathrm{e}^{\prime}\right)$ and Supplementary Table 2) also revealed similar results at the protein level of Bmil, SOD1, and SOD2 expression with the exception of Hsp70 expression after treatment with PTC-209. There were significant and marked differences between the PTC-209 and the control groups $\left(F=7931.17,{ }^{*} P<0.05\right)$. Moreover, RT-PCR showed that Bmil, SOD1, and SOD2 
(A)

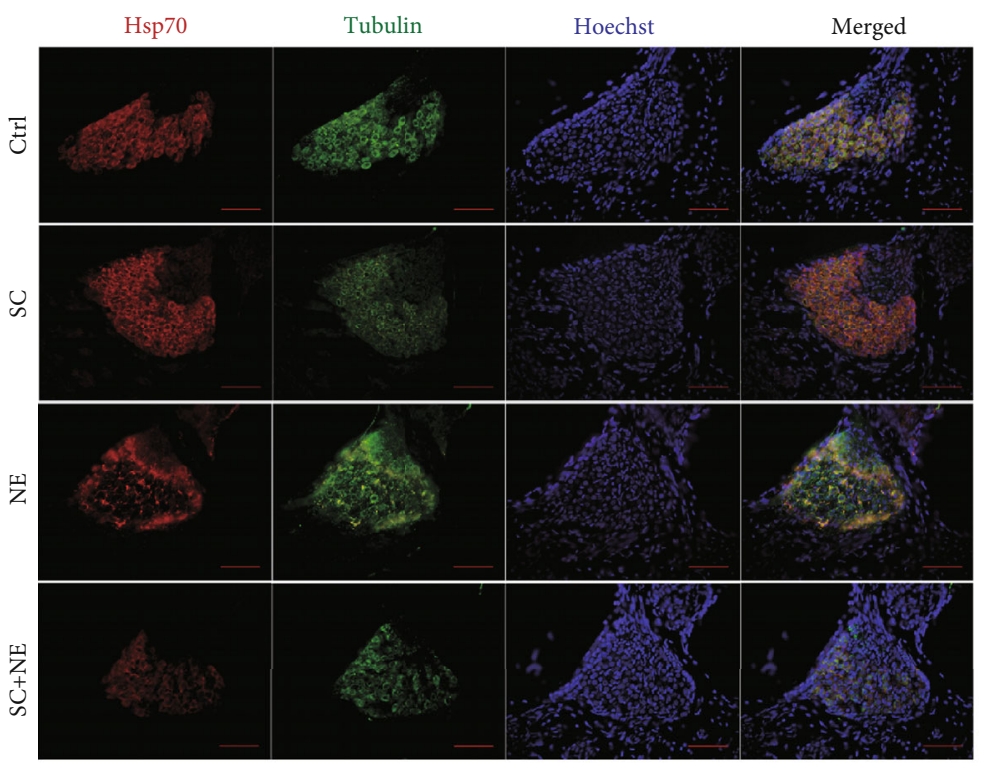

(a)

(A)

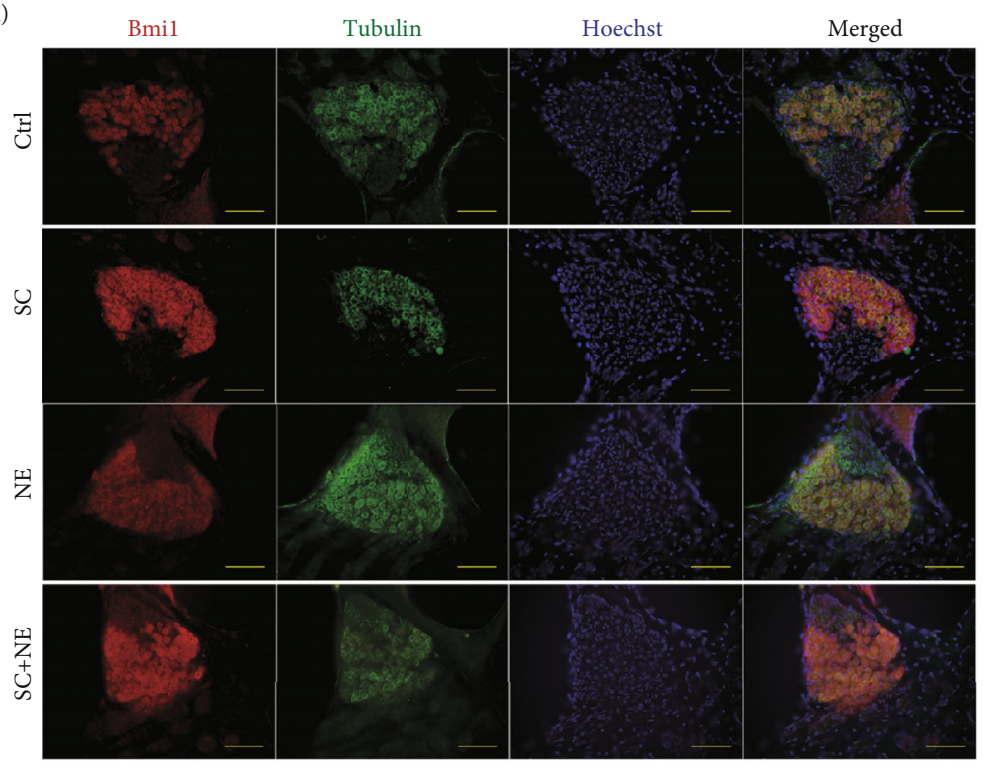

(B)

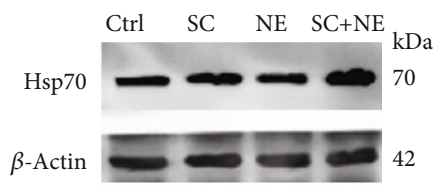

(C)

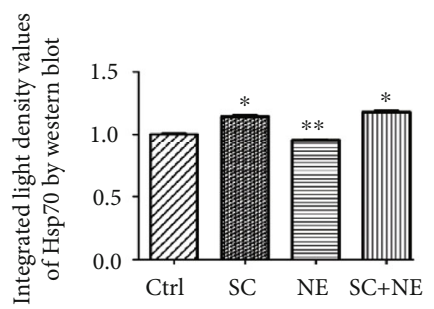

(D)

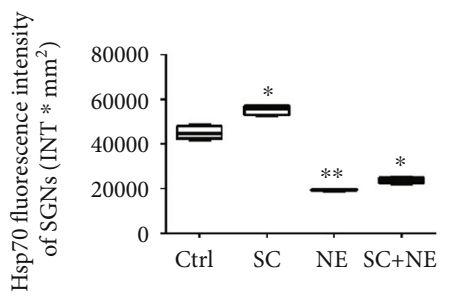

(B)

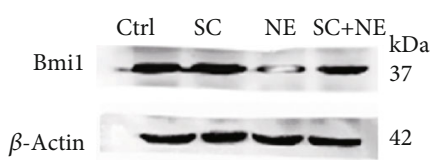

(C)

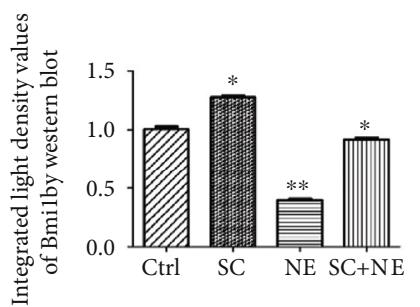

(D)

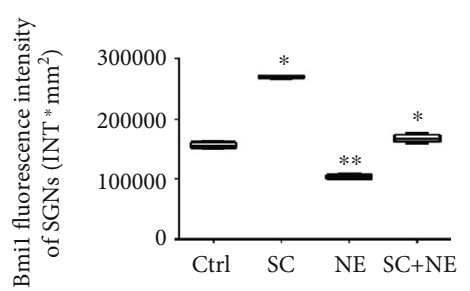

(b)

FIGURE 5: Hsp70 and Bmil protein expressions of SGNs after sound conditioning and acute noise exposure. Distributions and expressions of Hsp70 (a) and Bmil (b) protein were detected with the immune-fluorescence assay in the Ctrl and SC groups as well as the NE and SC+NE groups (A), while the quantity of these protein expressions was analyzed by fluorescence intensity (D). Hsp70/Bmil (red) and tubulin (green) as SGN marker were, respectively, labeled with fluorescent secondary antibody, and nuclei (blue) were labeled with Hoechst. Scale bars represent $100 \mu \mathrm{m}$. Protein expressions of these were also assessed with western blot (B), while the quantity of these protein expressions was analyzed by integrated light density $(\mathrm{C}) . \beta$-Actin protein was available as an endogenous control. Furthermore, values are means $\pm \mathrm{SD}$ ( $n=12$ animals/group). ${ }^{*} P<0.05$ vs. control group; ${ }^{* *} P<0.05$ vs. control group. Statistical analysis of the results presented in (C) was performed with one-way ANOVA (Hsp70: $F=126.5, P<0.0001$; Bmil: $F=478.8, P<0.0001$ ), followed by Newman-Keuls' post hoc test $\left({ }^{*} P<0.05,{ }^{* *} P<0.05 ; n=12\right.$ animals/group). Statistical analysis of the results presented in (D) was performed with one-way ANOVA (Hsp70: $F=303.4, P<0.0001$; Bmil: $F=940.7, P<0.0001$ ), followed by Newman-Keuls post hoc test $\left({ }^{*} P<0.05,{ }^{* *} P<0.05 ; n=6\right.$ pictures from 3 animals/group). Furthermore, values are means \pm SD. 


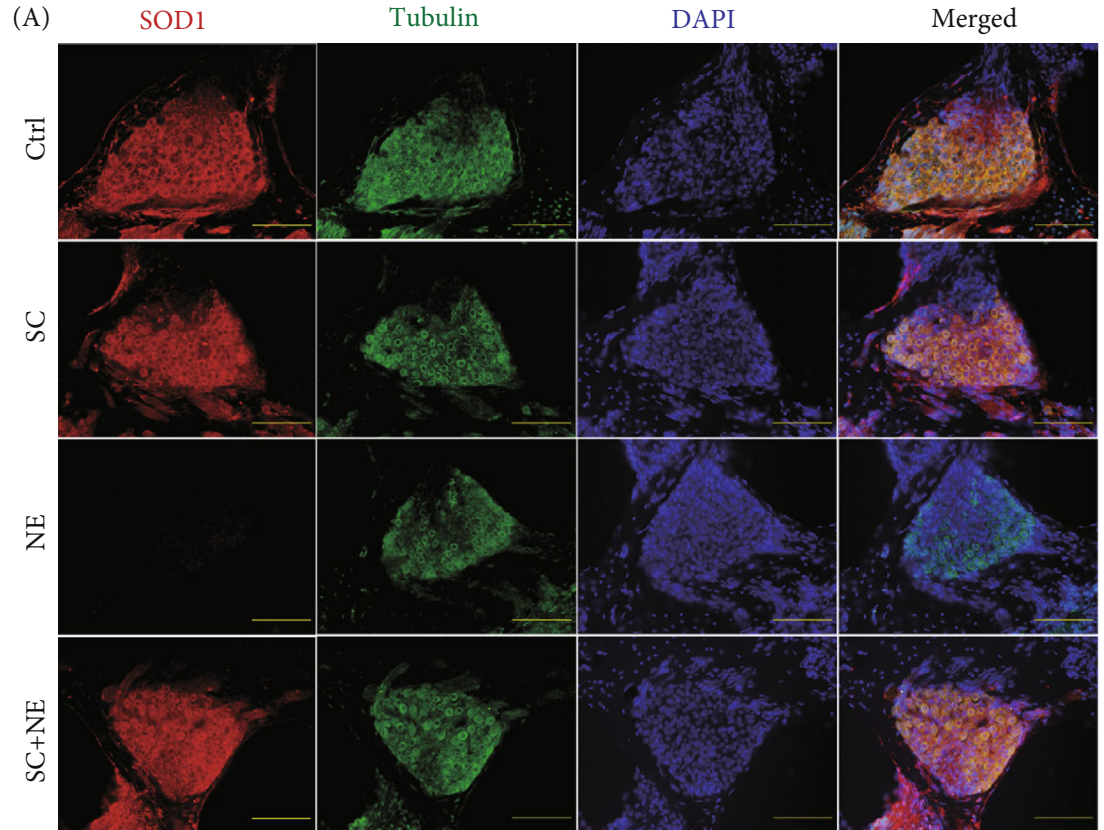

(a)

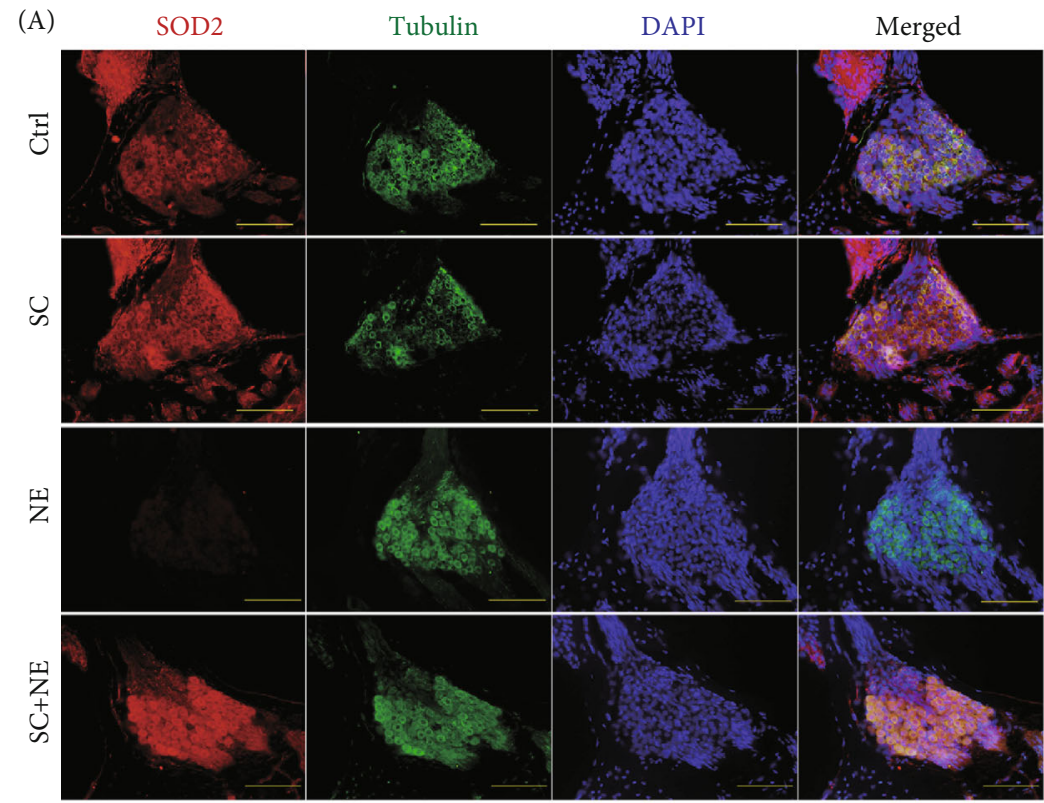

(B)

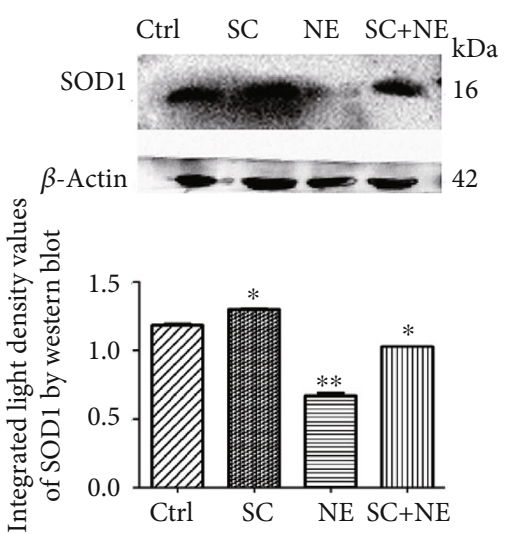

(D)

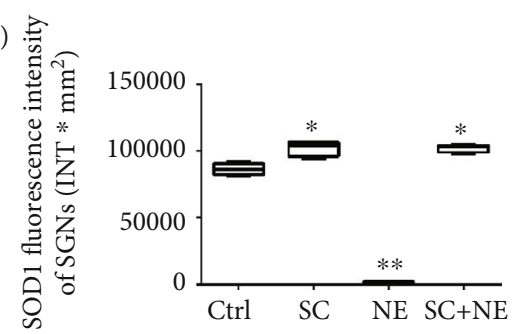

(B)

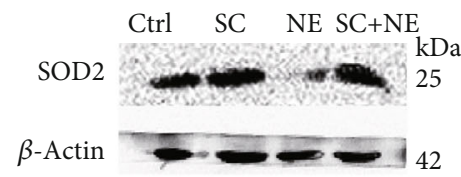

(C)
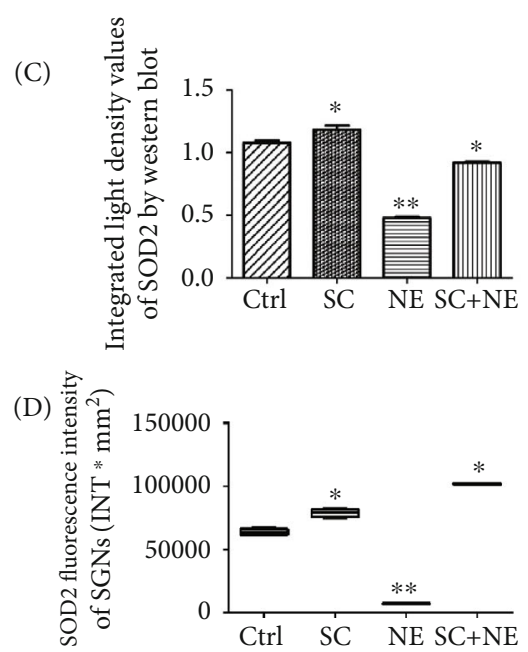

(b)

FIGURE 6: SOD1 and SOD2 protein expressions of SGNs after sound conditioning and acute noise exposure. Distributions and expressions of SOD1 (a) and SOD2 (b) protein were detected with the immune-fluorescence assay in the Ctrl and SC groups as well as the NE and SC+NE groups (A), while the quantity of these protein expressions was analyzed by fluorescence intensity (D). SOD1/SOD2 (red) and tubulin (green) as SGN marker were, respectively, labeled with fluorescent secondary antibody, and nuclei (blue) were labeled with DAPI. Scale bars represent $100 \mu \mathrm{m}$. Protein expressions of these were also assessed with western blot (B), while the quantity of these protein expressions was analyzed by integrated light density (C). $\beta$-Actin protein was available as an endogenous control. Furthermore, values are means $\pm \mathrm{SD}(n=12$ animals/group). ${ }^{*} P<0.05$ vs. control group; ${ }^{*} P<0.05$ vs. control group. Statistical analysis of the results presented in $(C)$ was performed with one-way ANOVA (SOD1: $F=579.5, P<0.0001$; SOD2: $F=271.3, P<0.0001$ ), followed by Newman-Keuls' post hoc test $\left({ }^{*} P<0.05\right.$, ${ }^{* *} P<0.05 ; n=12$ animals/group). Statistical analysis of the results presented in (D) was performed with one-way ANOVA (SOD1: $F=$ 1335.0, $P<0.0001$; SOD2: $F=1521, P<0.0001)$, followed by Newman-Keuls' post hoc test $\left({ }^{*} P<0.05,{ }^{* *} P<0.05 ; n=6\right.$ pictures from 3 animals/group). Furthermore, values are means \pm SD. 

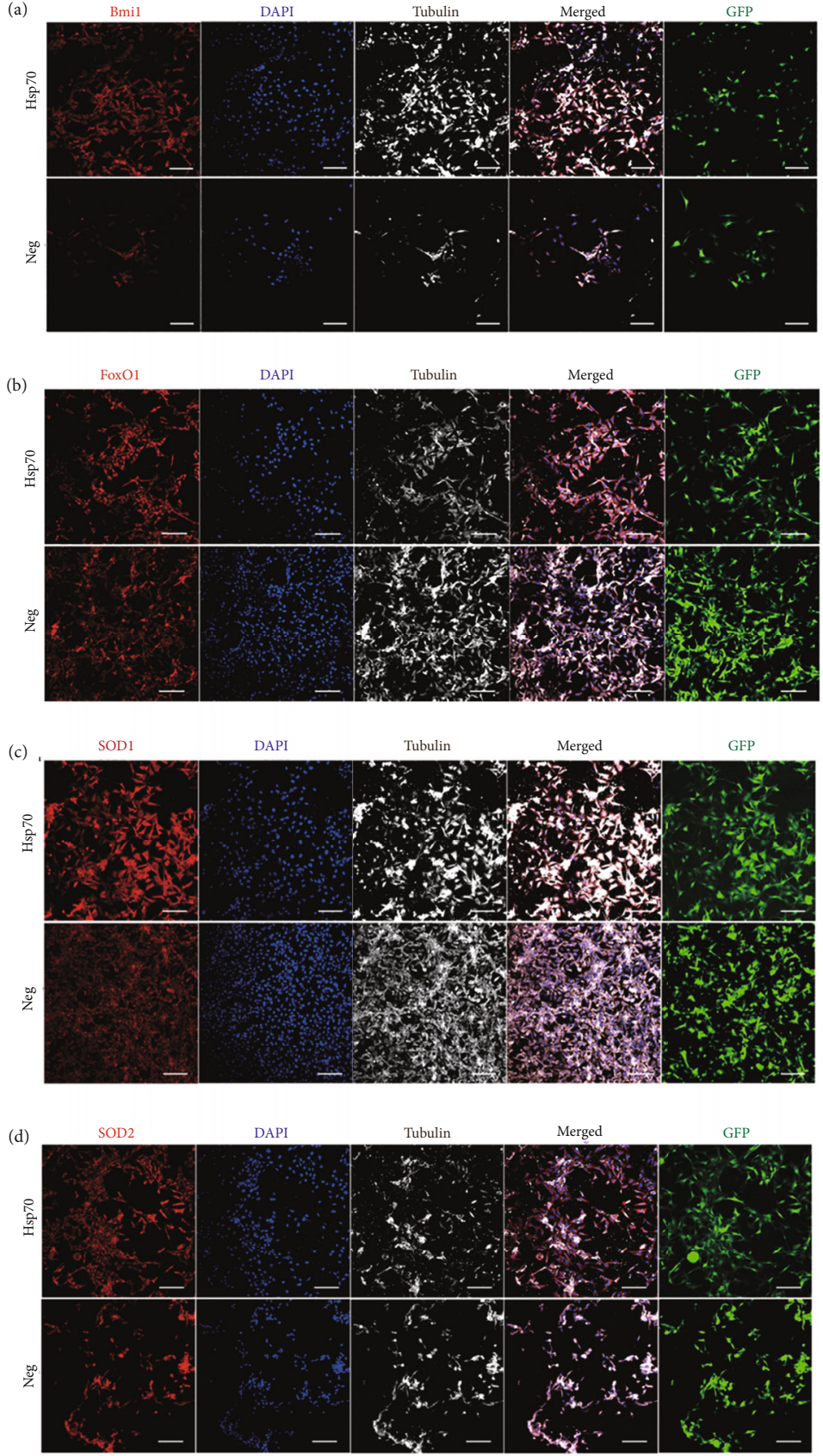
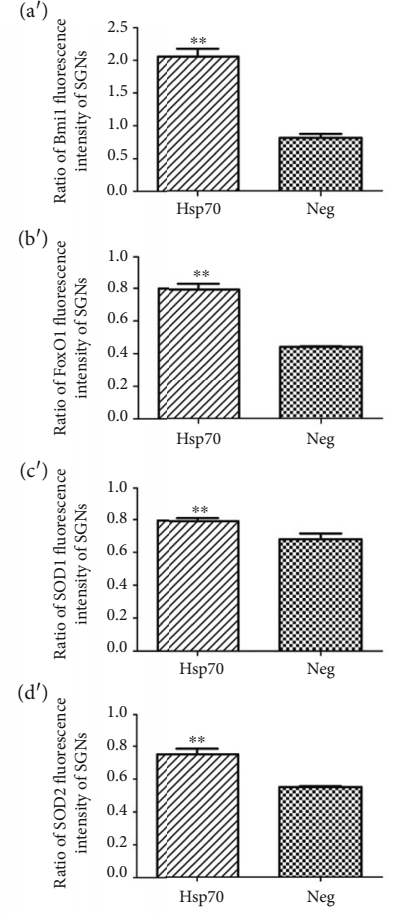

(e)
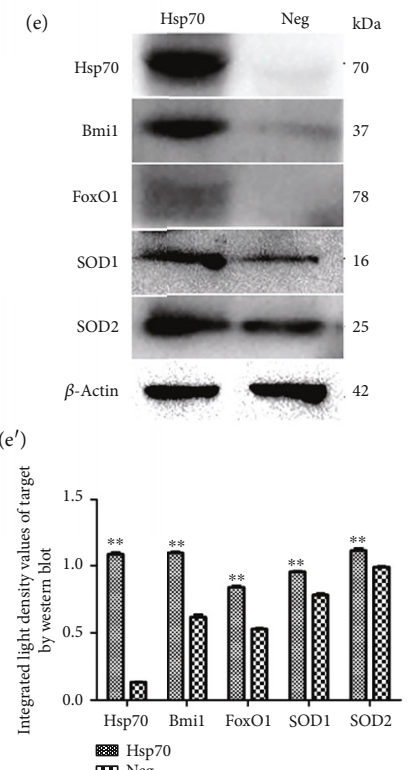

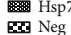

FIGURE 7: Bmi1, FoxO1, SOD1, and SOD2 expressions of overexpressed HSP70 SGNs. Distributions and expressions of Bmi1, FoxO1, SOD1, and SOD2 protein in SGNs were detected with the immune-fluorescence assay in the Hsp70-overexpressing adenovirus and negative control groups $(a-d)$, while the quantity of these protein expressions was analyzed by the ratio of fluorescence intensity $\left(a^{\prime}-d^{\prime}\right)$. After successful transfection by Hsp70-overexpressing adenovirus or empty vector adenovirus as GFP (green) shown, Bmi1/FoxO1/SOD1/SOD2 (red) and tubulin (white) as SGN marker were, respectively, labeled with fluorescent secondary antibody and nuclei (blue) were labeled with DAPI. Scale bars represent $100 \mu \mathrm{m}$. SGNs expressing a green fluorescent protein (GFP) coded in the same vector as the Hsp70-overexpressing vector was coupled to the GFP sequence, indicating that $66.7 \% \pm 7.8 \%(n=4$ independent cell culture preparations) of the neuronal cells present in the cultures were transfected with Hsp70. Therefore, ratios of Bmi1, FoxO1, SOD1, and SOD2 fluorescence intensity to GFP were calculated. Furthermore, values are means \pm SD $(N=40$ animals from 2 groups $) .{ }^{* *} P<0.05$ vs. negative group. Statistical analysis of the results presented in $\left(\mathrm{a}^{\prime}-\mathrm{d}^{\prime}\right)$ was performed with Student's $t$-test $\left({ }^{* *} P<0.05 ; N=40\right.$ animals from 2 groups). After successful transfection by Hsp70-overexpressing adenovirus or empty vector adenovirus, protein expressions of Hsp70, Bmi1, FoxO1, SOD1, and SOD2 were assessed with western blot (e), while the quantity of these protein expressions was analyzed by integrated light density ( $\mathrm{e}^{\prime}$ ). $\beta$ Actin protein was available as an endogenous control. Furthermore, values are means $\pm \mathrm{SD} .{ }^{* *} P<0.05$ vs. negative group. Statistical analysis of the results was performed with two-way ANOVA (interaction: $F(4,20)=459.8 ; P<0.0001 ;$ row factor: $F(4,20)=493.74 ; P<$ 0.0001 ; column factor: $F(1,20)=3400.31 ; P<0.0001)$, followed by Student's $t$-test $\left({ }^{* *} P<0.05 ; n=12\right.$ animals/group). 


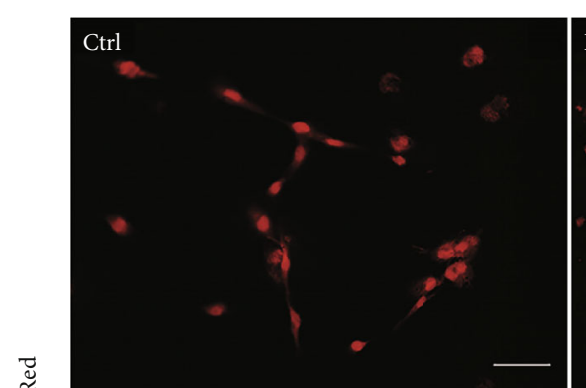

(a)

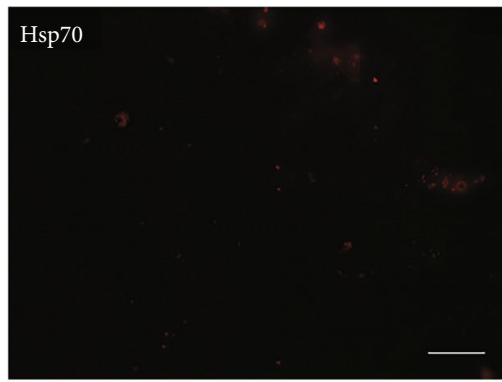

(c)

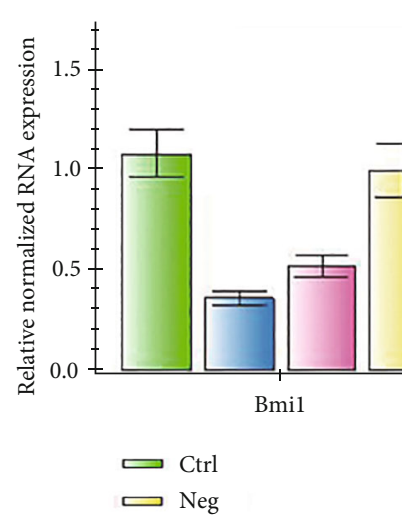

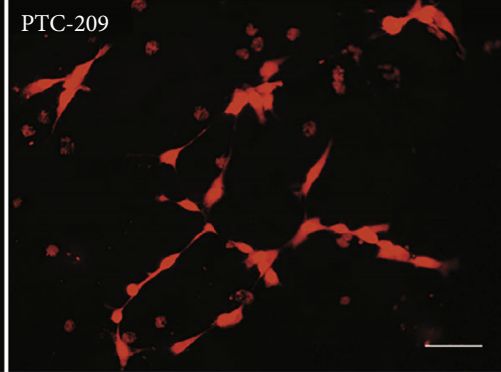

(b)

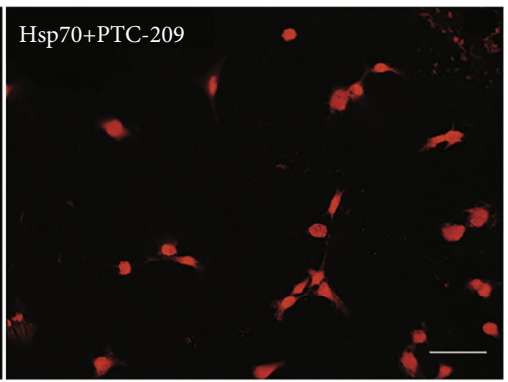

(d)

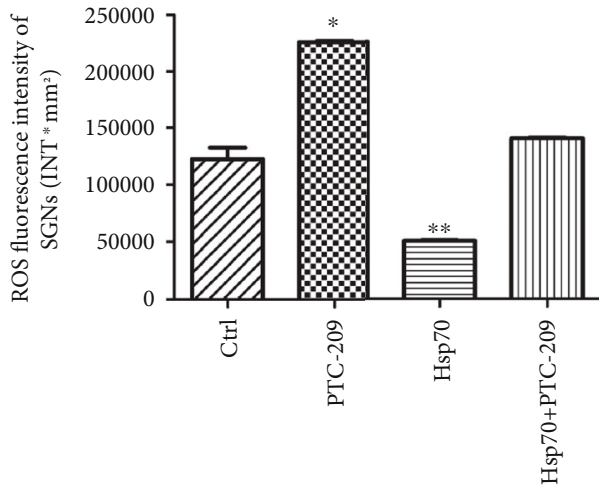

(e)

FIGURE 8: ROS accumulation of different treatments in SGNs and Bmil, Hsp70, SOD1, and SOD2 mRNA expression of SGNs in vitro. ROS accumulation in SGNs was showed by MitoSOX Red and was detected with the immune-fluorescence assay in the control group (a), PTC-209 treated group (b), Hsp70-overexpressing adenovirus group (c), and Hsp70-overexpressing adenovirus followed the PTC-209-treated group (d), while the quantity of ROS accumulation was analyzed by fluorescence intensity (e). Scale bars represent $50 \mu \mathrm{m}$. Furthermore, values are means $\pm \mathrm{SD}(N=80) .{ }^{*} P<0.05$ vs. control group; ${ }^{* *} P<0.05$ vs. control group. Statistical analysis of the results presented in (e) was performed with one-way ANOVA $(F=634.9 ; P<0.0001)$, followed by Newman-Keuls' post hoc test $\left({ }^{*} P<0.05,{ }^{* *} P<0.05 ; N=80\right.$ animals from 4 groups). Relative mRNA expressions of Bmi1, Hsp70, SOD1, and SOD2 were determined by quantitative RT-PCR (f). $\beta$ Actin RNA level was used as an endogenous control. Values are means $\pm \mathrm{SD}$ ( $n=12$ animals/group). ${ }^{*} P<0.05$ vs. control group.

mRNA expressions were also clearly decreased in the SGNs on neonatal cultures of the PTC-209 group compared with that of the control group except for Hsp70 mRNA expression (Figure 8(f)).

In addition, there was a significant difference between the PTC-209 and control groups in ROS accumulation of neonatal culture labeled by MitoSOX Red. In the PTC-209 group, ROS accumulation $\left(q=36.17,{ }^{*} P<0.05\right)$ was significantly increased in vitro, while a decrease in ROS accumulation of neonatal culture by the Hsp70-overexpressing adenovirus is offset by PTC-209 (Figures 8(a)-8(e)).
3.7. FoxO1 Was a Direct Target of Bmil in Rat SGNs. After treatment with PTC-209 on neonatal cultures in vitro, IF results (Figure $9(\mathrm{~b}) / 9\left(\mathrm{~b}^{\prime}\right)$ ) revealed that FoxO1 (a transcription factor responsible for the stress response and redox balance) fluorescence levels $\left(t=13.02,{ }^{*} P<0.05\right)$ are lower in the SGNs. WB analysis (Figure $9(\mathrm{e}) / 9\left(\mathrm{e}^{\prime}\right)$ ) also showed that FoxO1 expression $\left(t=50.89,{ }^{*} P<0.05\right)$ is decreased in SGNs on neonatal cultures of the PTC-209 group. There was a significant difference found between the PTC-209 and control groups in FoxO1 expression $\left(F=7931.17,{ }^{*} P<0.05\right)$, which was directly decreased by PTC-209. 
(a)

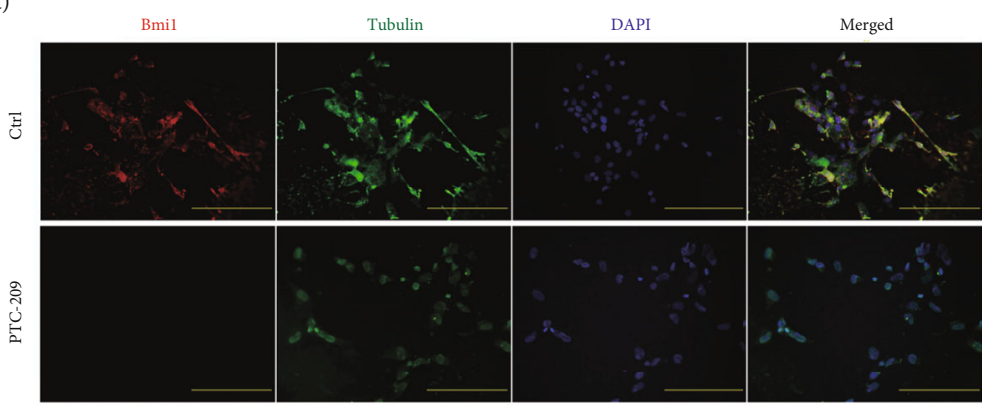

(b)

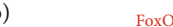

FoxO

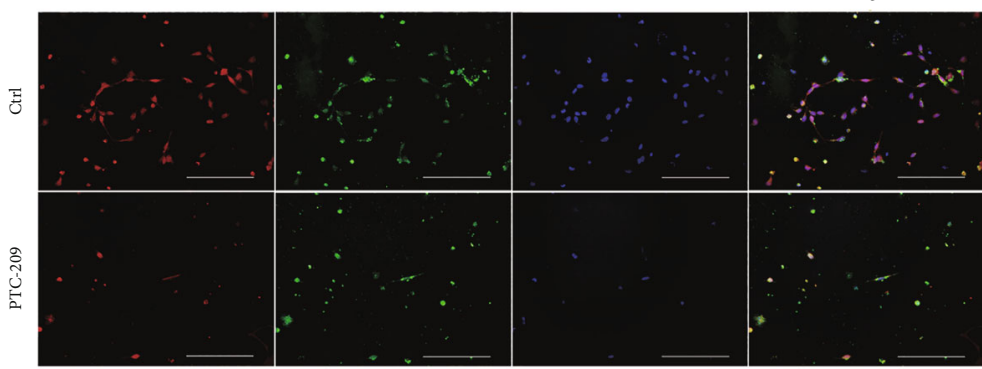

(c)

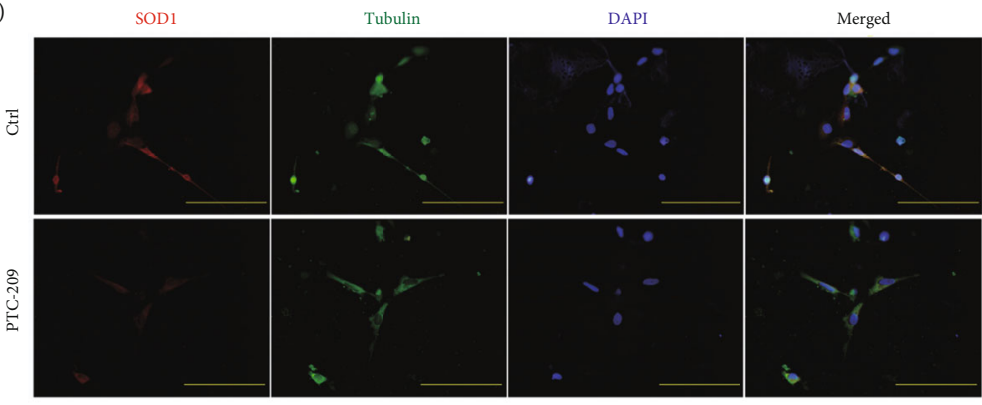

(d)

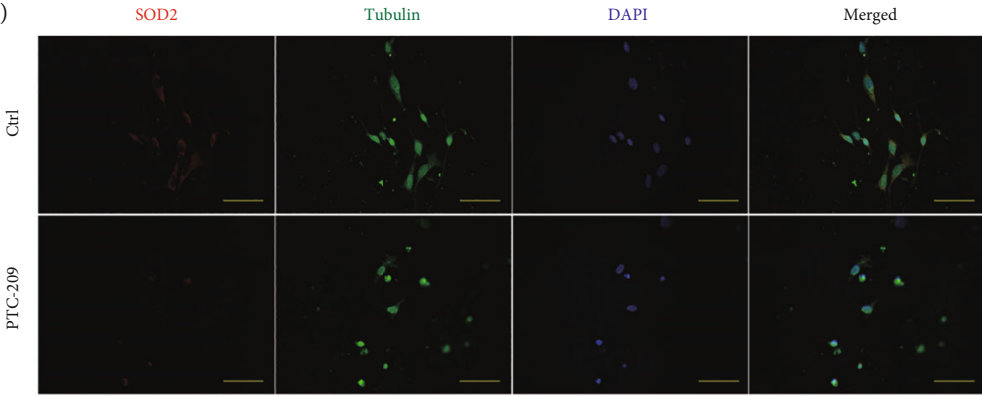

$\left(\mathrm{a}^{\prime}\right)$

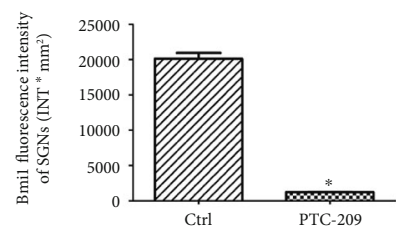

$\left(b^{\prime}\right)$

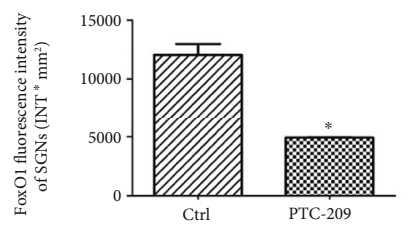

(c')

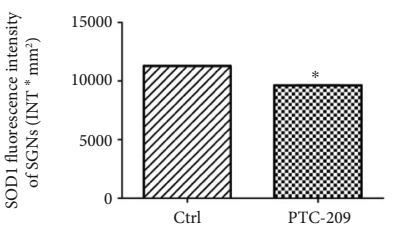

$\left(d^{\prime}\right)$

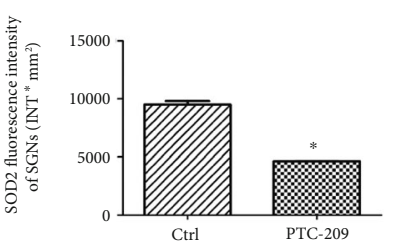

(e)

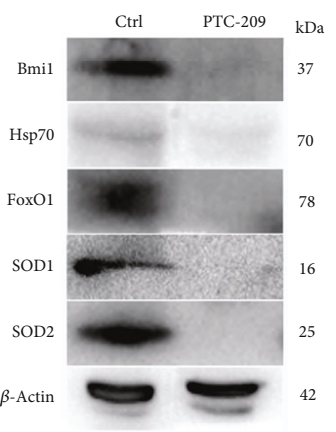

$\left(\mathrm{e}^{\prime}\right)$

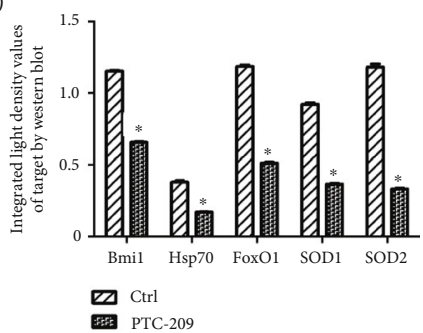

FIGURE 9: Bmil, FoxO1, SOD1, and SOD2 expressions in SGNs after PTC-209 treatment. Distributions and expressions of Bmil, FoxO1, SOD1, and SOD2 protein in SGNs were detected with the immune-fluorescence assay in the control group and the PTC-209 treated groups $(a-d)$, while the quantity of these protein expressions was analyzed by fluorescence intensity $\left(a^{\prime}-d^{\prime}\right)$. After PTC-209 treatment, Bmil/FoxO1/SOD1/SOD2 (red) and tubulin (green) as SGN marker were, respectively, labeled with fluorescent secondary antibody and nuclei (blue) were labeled with DAPI. Scale bars represent $100 \mu \mathrm{m}$ and $50 \mu \mathrm{m}$. Furthermore, values are means \pm SD $(N=40$ animals from 2 groups). ${ }^{*} P<0.05$ vs. control group. Statistical analysis of the results presented in $\left(\mathrm{a}^{\prime}-\mathrm{d}^{\prime}\right)$ was performed with Student's $t$-test $\left({ }^{*} P<0.05\right.$ ; $N=40$ animals from 2 groups). After PTC-209 treatment, protein expressions of Hsp70, Bmil, FoxO1, SOD1, and SOD2 were assessed with western blot (e), while the quantity of these protein expressions was analyzed by integrated light density $\left(\mathrm{e}^{\prime}\right)$. $\beta$-Actin protein was available as an endogenous control. Furthermore, values are means \pm SD. ${ }^{*} P<0.05$ vs. control group. Statistical analysis of the results was performed with two-way ANOVA (interaction: $F(4,20)=283.78 ; P<0.0001$; row factor: $F(4,20)=1276.15 ; P<0.0001$; column factor: $F$ $(1,20)=7931.17 ; P<0.0001)$, followed by Student's $t$-test $\left({ }^{*} P<0.05 ; n=12\right.$ animals/group). 


\section{Discussion}

Hearing loss is a common sensory disorder all over the world; by far, there are around 466 million patients with hearing disability worldwide. In order to have hearing, HCs are needed for transducing sound vibrations into electrical signaling, and SGNs transmit these electrical signals into the auditory cortex [29-32]. Thus, the majority of the hearing loss is caused by the malfunction or damage of HCs or SGNs. Both HCs or SGNs are vulnerable to be injured by noise, ototoxic drugs, inflammation, biological aging, and genetic defects [16, 33-37]. In recent years, many previous reports used transcription regulation to promote the inner ear stem cells to regenerate the HCs [38-42] and used biomaterials, electrical stimulation, and magnetic regulation to promote the neural stem cells to regenerate the SGNs [43-46] and to promote the maturation of SGNs [47-49]. However, the mammals only have very limited $\mathrm{HC}$ and SGN regeneration ability [50-55]; thus, hearing loss is irreversible by far. Thus, to fully understand the detailed protective mechanism of SC in mammals and to effectively protect the HCs and SGNs against various damages are very important. The primary goals of this study were to identify the involvement of Hsp70 and Bmil in the protective effect of SC against AAT by targeting SOD1 and SOD2, regulated by the FoxO1 signaling pathway, and to verify the effects of free oxygen radical alteration related to the underlying mechanisms in vitro and in vivo. In this study, we confirmed that SC prior to a loud noise protected against AAT based on significant improvements in hearing impairment and an apparent reduction in $\mathrm{OHC}$ loss (Figure 2) as well as ultrastructural changes in OHCs and SGNs of a fully mature rat (Figure 3). Next, we reported that these functional and ultrastructural changes in rat SGNs were accompanied by significant upregulation of Hsp70, Bmi1, FoxO1, SOD1, and SOD2 protein expression (Figures 5, 6, and 4(a)), which indicated that these proteins were involved in the protective effect of SC against AAT in rats. Furthermore, we used transfection with Hsp70-overexpressing adenovirus and coculture with PTC-209 to verify the Hsp70/Bmil-FoxO1-SOD signaling pathway and its effect on ROS (mitochondrial superoxide) accumulation of neonatal culture in vitro (Figures 7-10). To our knowledge, this is the first report that upregulation of Hsp70 and Bmil expression was involved in the protective effect of SC against AAT by targeting SOD1 and SOD2 in rat SGNs, regulated by the FoxO1 signaling pathway.

Previous research lends progressive support to the evidence that oxidative stress, generated in part by glutamate excitotoxicity, impaired mitochondrial function, and GSH depletion resulted in cochlear injury induced by AAT. Therefore, enhancing the cellular oxidative stress defense pathways in the cochlea alleviated noise-induced cochlear injury [56]. Previous studies have further confirmed that AAT is closely related to the degeneration of the antioxidant system in the organs [11-14]. Thus, SC could protect hearing by enhancement of the antioxidant system [1-5, 7, 57-59]. Our results in vivo also indicated that SC protected against AAT as it did not result in hearing loss in the studied rats. Furthermore, hair cell loss caused by NE was reduced by SC in both the base and middle turns of the basilar membrane, while the apex is less vulnerable to AAT and SC is of no advantage in that area. Interestingly, SC also increased the quantity of mitochondria, enhanced their function in the SGN cells, and reversed the injury and dysfunction of mitochondria in the SGNs caused by NE. The changes in mitochondria of SGNs were direct proof that the antioxidant system in the organs was enhanced, which contributed to less sensitivity in AAT. Therefore, the findings above in our study fully supported the suggestion that SC could protect against AAT by enhancement of the antioxidant system in the SGNs. Moreover, SC was also reported to be capable of improving the removal of oxidation products by enhancement of the antioxidant system, which protected against AAT [3]. Furthermore, we found that Hsp70, Bmil, FoxO1, SOD1, and SOD2 were all involved in the SC-induced enhancement of the antioxidant system and reduced ROS accumulation in the SGNs.

It is widely known that the Hsp70 family of heat shock proteins composed of molecular chaperones of approximately $70 \mathrm{kDa}$ in size played a critical role in protein homeostasis. The Hsp70 proteins possess a highly conserved domain structure that is comprised of the following main domains: an $\sim 44 \mathrm{kDa}$-terminal nucleotide-binding domain (NBD) which exhibited ATPase activity and was highly conserved; a middle flexible linker region; an $\sim 15 \mathrm{kDa}$ substrate-binding domain (SBD), which interacts with the stretches of the hydrophobic amino acids in the peptides; and an $\sim 10 \mathrm{kDa} \alpha$-helical C-terminal domain that is believed to form a "lid" that closes over the substrate and mediated cochaperone binding [60-63]. Hsp70 proteins played a crucial role in the mediation of correct protein folding and, consequently, in the maintenance of protein homeostasis. Hsp70 directly unfolded misfolded proteins, in adenosine triphosphate- (ATP-) dependent fashion. These proteins also enhanced cell survival following a multitude of stressors, including elevated temperature, hypoxia, oxidative stress, and others. This survival role was reflected in the ability of Hsp70 to buffer the toxicity of denatured and misfolded proteins that accumulated during stress [19]. Many previous studies demonstrated that Hsp induction was a critical stress response in the inner ear that could promote the survival of hair cells exposed to both classes of ototoxic drugs [64-68] and lowered the risks of AAT in mice [69]. In addition, the expression of Hsp70 was increased in the inner ear of mice by exposure to low-frequency noise (LFN), which suggested that Hsp70 played an important part in protecting the inner ears from LFN [70]. Furthermore, strong evidence from recent studies has also shown that SC triggered Hsp70 induction and enhanced its expression in vivo $[5,7,59,71-73]$. According to our study, the RT-PCR, WB, and IF results showed that increased expression of Hsp70 was also involved in the protective effects of SC against AAT in rats. Therefore, we suggested that SC increased Hsp70 expression in the SGNs to enhance cell survival following a noise-induced stress response.

Conversely, evidence from previous studies suggested that Bmil improved cell survival by regulating mitochondrial function and ROS levels in the thymocytes and neurons, 


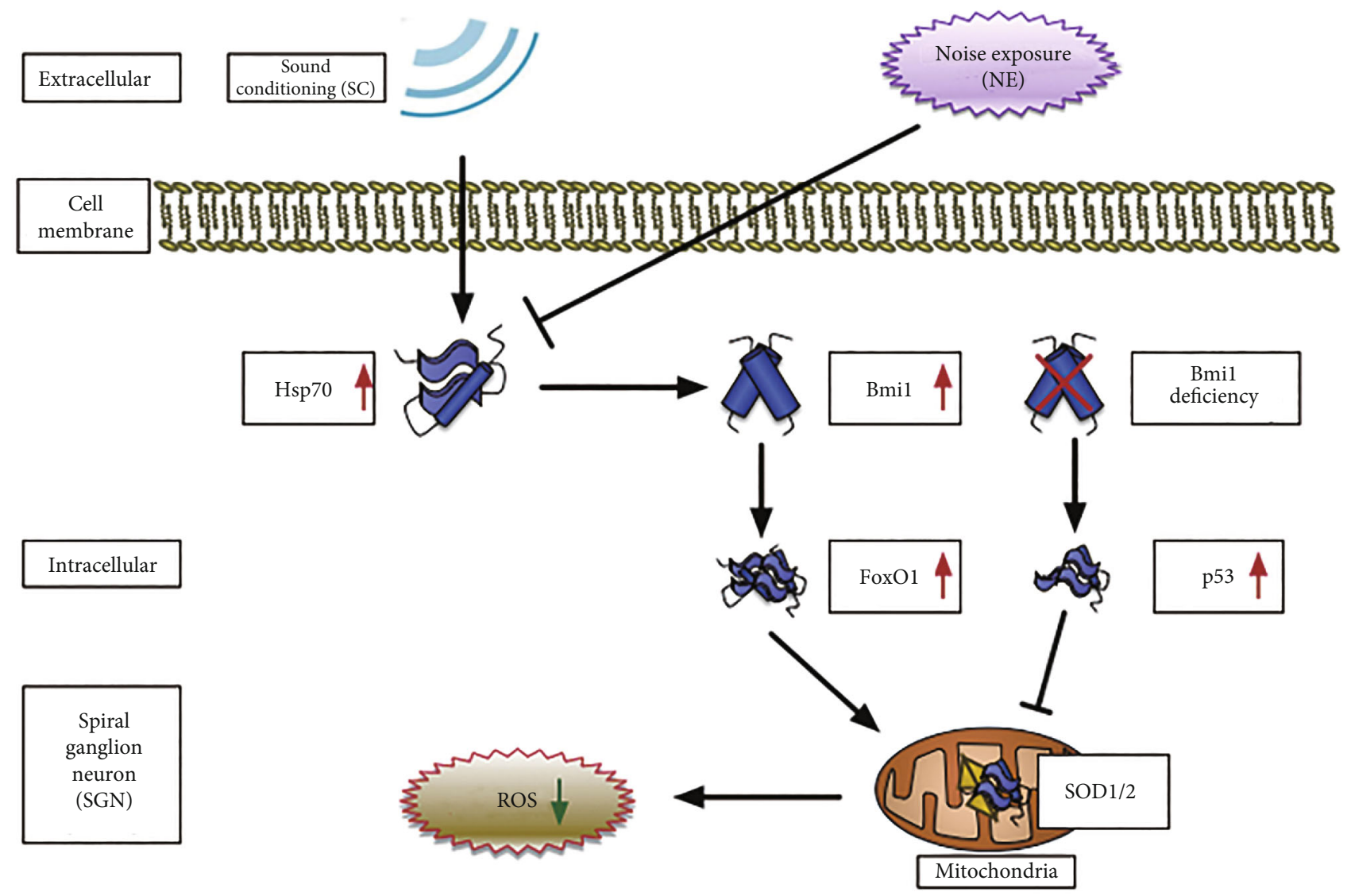

Figure 10: A model for the mechanism of Hsp70-induced Bmil upregulation in the protection of sound conditioning against acute acoustic trauma by directly targeting SOD1 and SOD2 regulated by the FoxO1 signaling pathway. Hsp70/Bmi1-FoxO1-SOD1/SOD2 signaling pathway decreases sensitivity in noise-induced trauma following sound conditioning by controlling mitochondrial function and ROS level in rat's SGN. Meanwhile, based on previous studies, other pathway is also involved in Bmil deficiency-induced ROS accumulation, such as the following: Bmil deficiency directly leads to p53-mediated repression of antioxidant genes, resulting in increased ROS.

which had a close bearing on the antioxidants and the removal of oxidation products [3]. Bmil, a member of the polycomb protein family, could bind to the Runx1/CBF $\beta$ transcription factor complex to silence target genes in a PRC2-independent manner [74]. Abdouh et al. confirmed that other than in stem cells and rapidly dividing cells, Bmil was expressed in terminally differentiated cells such as neurons and increased in Bmil expression in cortical neurons which activated antioxidant genes to regulate ROS generation and protected against DNA damage-induced cell apoptosis as well as mitochondrial injury [75]. In neurons, Bmil also suppressed p53-induced cell apoptosis by regulating antioxidation [76]. Furthermore, a previous report also showed that Bmil-deficient thymocytes impaired mitochondrial function, which led to a marked increase in intracellular ROS levels and a subsequent engagement of the DDR pathway [77]. Rizo et al. found that a reduced ability of self-renewal was associated with enhanced apoptosis in Bmil- CD34(+) stem cells, which coincided with increased levels of intracellular ROS [78]. Furthermore, overexpression of Bmil in vivo protected human embryonic stem cells (HSCs) from ROS damage and extended the lifespan of HSCs [79], whereas Bmil transduction in vitro reduced irradiation-induced ROS levels by suppressing the oxidase genes and increased repair of the DNA damage in human keratinocytes [80]. Furthermore, in a recent study by Chen et al., Bmil was demonstrated to have an important role in hair cell survival via controlling the redox balance and ROS levels [20]. Our study in vivo and in vitro produced similar results to Chen et al. However, the current study also found that these functional and ultrastructural changes of mitochondria were accompanied by significant upregulation of Hsp70 and Bmil expression induced by SC and that overexpression of Hsp70 significantly enhanced Bmil expression in rat SGNs (Hsp70 might stabilize Bmil at the protein level rather than increase it at the transcriptional level) and clearly decreased mitochondrial superoxide accumulation, while Bmil inhibitor attenuated the effect of Hsp70 transfection. This finding suggested that SC induce increased Bmil expression in the SGNs, which was triggered by Hsp70 induction to improve cell survival by regulating mitochondrial function and mitochondrial superoxide levels.

It is widely accepted that several pathways are involved in Bmil deficiency-induced ROS accumulation, including the following: (1) Bmil deficiency leads to mitochondrial dysfunction resulting in a rise of intracellular ROS and subsequent engagement of the DDR pathway, in which Chk2 and 
p53 were activated [77]; (2) Bmil deficiency directly leads to p53-mediated repression of the antioxidant genes, resulting in increased ROS levels [75]. However, how to control mitochondrial function and ROS level in SGNs by Bmil upregulation and the mechanism by which SC exerted a protective effect against AAT have remained unknown. To fix this problem, we further investigated FoxO1 (a transcription factor responsible for the stress response and redox balance) [81], SOD1 (an antioxidant gene whose protein is found in the cytoplasm), and SOD2 (an antioxidant gene whose protein is found in the mitochondrial ridge) expressions in SGNs in vivo and in vitro by transfection with Hsp70-overexpressing adenovirus and coculture with PTC209. Ultimately, we found that functional and ultrastructural changes in the mitochondria were still associated with a significant upregulation of FoxO1, SOD1, and SOD2 expression induced by SC and we also confirmed that PTC-209 significantly decreased FoxO1, SOD1, and SOD2 expressions in the rat SGNs and increased mitochondrial superoxide accumulation, while overexpression of $\mathrm{Hsp} 70$ significantly reversed these expressions in the rat SGNs. We suggested that changes in SOD1/SOD2 expression could be involved in the protective effects of SC and a consequence of cell protection. Therefore, our results indicated that the Hsp70/Bmil-FoxO1-SOD signaling pathway was involved in the protective effect of SC against AAT and regulated mitochondrial function and the mitochondrial superoxide levels in rat SGNs (Figure 10), which coincided with the regulation of oxidative stress and mitochondrial dysfunction by FoxO1 in human QBC939 cells from a recent study [82]. Meanwhile, the involvement of the Hsp70/Bmil-FoxO1SOD signaling pathway in other regions of cochlear tissue (such as hair cells and cochlear lateral wall) behind the protective effect of SC has also been conformed further by our immunolabeling data. However, in view of the complexity of regulating Bim1 expression in SGNs, we still believe that there might be additional signaling pathways that regulate mitochondrial function and ROS levels in rat SGNs. Therefore, more research on this issue should be performed. In addition, the overexpression experiments on cultured SGN were performed on neonatal cultures whereas our noise exposure/preconditioning experiments were performed on fully mature rats. Although immature SGNs differ in many ways from mature ones (expression of ion channels, etc.) [83], there is no significant difference between the changes of a target molecule in vivo and in vitro based on our current research data.

Thus, we found that SC of low frequency prior to a loud noise not only protected against AAT based on significant improvements in hearing impairment and an apparent reduction in OHC loss but also improved SGN survival following a noise-induced stress response via controlling mitochondrial function and ROS levels in rat SGNs, and we are the first to have demonstrated a new theory on the protection of SC against AAT in which the Hsp70/BmilFoxO1-SOD signaling pathway was involved. Lastly, we suggested that the enhancement of the antioxidant system and a reduction in ROS accumulation might be the main reasons for decreased sensitivity to noise-induced trauma following treatment with SC. The results from our work help us to understand the protective capacity of SC against AAT and the underlying mechanisms involved.

\section{Conclusions and Future Directions}

In summary, the in vitro and in vivo data presented here provide evidence that (i) SC improved SGN survival following noise-induced stress response via controlling mitochondrial function and ROS levels, (ii) Hsp70/Bmil-FoxO1-SOD signaling pathway was involved in the protection of SC against $\mathrm{AAT}$, and (iii) enhancement of the antioxidant system and a reduction in ROS accumulation might be the main reasons for the decreased sensitivity to AAT following treatment with SC in the rats. Clinically, these issues could provide a better preventive strategy for AAT.

5.1. Limitations of Our Work. Due to technical issues, the harvested tissues in western blot included other supporting cells in addition to SGN so that the data of western blot does not coincide with that of immunofluorescence in vivo (especially for Hsp70). This issue might be resolved by tissue purification technology. The roles of Hsp70 and Bmil have been demonstrated in vitro but not fully in vivo. The role of FoxO1 in the upregulation of SOD should be demonstrated further. Furthermore, so far, it is still unclear how the FoxO1 signaling pathway activates antioxidant genes to control mitochondrial function and ROS levels. Finally, whether the SC stimulus of other higher frequencies or even noise might protect against hearing loss more effectively or not should be considered to study further. Therefore, more research on these issues should be performed in our next study.

\section{Data Availability}

The data used to support the findings of this study were supplied by Jianhua Qiu under license and so cannot be made freely available. Requests for access to these data should be made to Jianhua Qiu (qiujh@fmmu.edu.cn).

\section{Conflicts of Interest}

The authors declare no competing interests.

\section{Authors' Contributions}

ZGX, WYX, and QY proposed the idea, did the research, analyzed data, and wrote the paper; TKY, MWJ, LXQ, CYY, and JJW provided support and participated in discussions on the research; QJH, LLJ, and LJS gave us valuable advice and revised the manuscript. All authors have read and approved the final manuscript. Guoxia Zhu, Yongxiang $\mathrm{Wu}$, and Yang Qiu contributed equally to this work.

\section{Acknowledgments}

The authors wish to thank Zhenzhen Liu for excellent technical assistance. We thank American Journal Experts for editing service. This study was supported by grants from the International Cooperation and Communication Project of the 
Natural Scientific Foundation of China (NSFC, No. 81110097), the Natural Scientific Foundation of China (NSFC, Nos. 81400459, 81172635, and 81803187), the Youth Development Project of Military Medical Science and Technology (MMST, Nos. 14QNP023), and the Scientific Incubation Foundation of Xi'an People's Hospital/Xi'an Fourth Hospital (Nos. ZD-3).

\section{Supplementary Materials}

Supplementary 1. Supplementary Figure 1: Hsp70 expression in SGNs after PTC-209 treatment distribution and expression of Hsp70 protein in SGNs was detected with immunofluorescence assay in the control group and PTC-209 treated groups (A), while the quantity of $\mathrm{Hsp} 70$ protein expression was analyzed by fluorescence intensity (B). After PTC-209 treatment, Hsp70 (red) and tubulin (green) as SGN marker were, respectively, labeled with fluorescent secondary antibody and nuclei (blue) were labeled with DAPI. Scale bars represent $50 \mu \mathrm{m}$. Furthermore, values are means \pm SD ( $N=40$ animals from 2 groups). $P>0.05$ vs. control group. Statistical analysis of the results presented in (B) was performed with Student's $t$-test $(P>0.05 ; N=40$ animals from 2 groups). Supplementary 2. Supplementary Figure 2: Hsp70, SOD1, SOD2, and Bmil mRNA expression of SGNs in vitro. Relative mRNA expressions of Hsp70, SOD1, SOD2, and Bmil were determined by quantitative RT-PCR. $\beta$-Actin RNA level was used as an endogenous control. Values are means $\pm \mathrm{SD}\left(n=12\right.$ animals/group). ${ }^{*} P$ $<0.05 \mathrm{Hsp} 70$ vs. control group. Supplementary 3. Supplementary Figure 3: Hsp70 and Bmil protein expressions in hair cells and cochlear lateral wall after sound conditioning and acute noise exposure. Distributions and expressions of Hsp70 (A) and Bmil (B) protein were detected with immunofluorescence assay in the Ctrl and SC groups as well as the NE and SC+NE groups (a), while the quantity of these protein expressions in both hair cells (b) and cochlear lateral wall (c) was analyzed by fluorescence intensity. Hsp70/Bmil (red) was labeled with fluorescent secondary antibody, and nuclei (blue) were labeled with Hoechst. Scale bars represent $100 \mu \mathrm{m}$. Furthermore, values are means \pm SD. Statistical analysis of the results presented in (b) (Hsp70: $F=80.74, P$ <0.0001; Bmil: $F=86.64, P<0.0001)$ and (c) $($ Hsp70: $F=$ 684.6, $P<0.0001$; Bmil: $F=338.2, \quad P<0.0001)$ was performed with one-way ANOVA, followed by NewmanKeuls' post hoc test $\left({ }^{*} P<0.05,{ }^{* *} P<0.05 ; n=6\right.$ pictures from 3 animals/group). Supplementary 4 . Supplementary Figure 4 : SOD1 and SOD2 protein expressions in hair cells and cochlear lateral wall after sound conditioning and acute noise exposure. Distributions and expressions of SOD1 (A) and SOD2 (B) protein were detected with immunofluorescence assay in the Ctrl and SC groups as well as the NE and SC+NE groups (a), while the quantity of these protein expressions in both hair cells (b) and cochlear lateral wall (c) was analyzed by fluorescence intensity. SOD1/SOD2 (red) was labeled with fluorescent secondary antibody, and nuclei (blue) were labeled with Hoechst. Scale bars represent $100 \mu \mathrm{m}$. Furthermore, values are means \pm SD. Statistical analysis of the results presented in (b) (SOD1: $F=233.0, P<0.0001$; SOD2: $F=79.93, P<0.0001$ ) and (c) (SOD1: $F=143.0, P<0.0001$; SOD2: $F=199.8, P<$ $0.0001)$ was performed with one-way ANOVA, followed by Newman-Keuls' post hoc test $\left({ }^{*} P<0.05,{ }^{* *} P<0.05 ; n=6\right.$ pictures from 3 animals/group). Supplementary 5 . Supplementary Table 1: statistical results of immunofluorescence of target protein expression in vivo and in vitro (Student-Newman-Keuls(SNK-) $q$ test for post hoc comparisons (NE or SC vs. Ctrl) ${ }^{* *} P<0.05,{ }^{*} P<0.05$; Student's $t$-test for the comparisons between the two groups (Hsp70 vs. Neg or PTC-209 vs. Ctrl) $\left.{ }^{* *} P<0.05,{ }^{*} P<0.05\right)$. Supplementary 6 . Supplementary Table 2: statistical results of western blot of target protein expression in vivo and in vitro (Student-Newman-Keuls- (SNK-) $q$ test for post hoc comparisons (NE or SC vs. Ctrl) ${ }^{* *} P<0.05,{ }^{*} P<0.05$ ; Student's $t$-test for the comparisons between the two groups (Hsp70 vs. Neg or PTC-209 vs. Ctrl) ${ }^{* *} P<0.05,{ }^{*} P<0.05$ ). (Supplementary Materials)

\section{References}

[1] T. Miyakita, P. A. Hellstrom, E. Frimanson, and A. Axelsson, "Effect of low level acoustic stimulation on temporary threshold shift in young humans," Hearing Research, vol. 60, no. 2, pp. 149-155, 1992.

[2] M. Pukkila, S. Zhai, J. Virkkala, U. Pirvola, and J. Ylikoski, "The "toughening" phenomenon in rat's auditory organ," Acta Oto-Laryngologica. Supplementum, vol. 529, pp. 59-62, 1997.

[3] A. A. Jacono, B. Hu, R. D. Kopke, D. Henderson, T. R. Van De Water, and H. M. Steinman, "Changes in cochlear antioxidant enzyme activity after sound conditioning and noise exposure in the chinchilla," Hearing Research, vol. 117, no. 1-2, pp. 31-38, 1998.

[4] X. Niu and B. Canlon, "Activation of tyrosine hydroxylase in the lateral efferent terminals by sound conditioning," Hearing Research, vol. 174, no. 1-2, pp. 124-132, 2002.

[5] H. Zuo, B. Cui, X. She, and M. Wu, "Changes in guinea pig cochlear hair cells after sound conditioning and noise exposure," Journal of Occupational Health, vol. 50, no. 5, pp. 373379, 2008.

[6] J. C. Alvarado, V. Fuentes-Santamaria, M. C. Gabaldon-Ull, T. Jareno-Flores, J. M. Miller, and J. M. Juiz, "Noise-induced "toughening" effect in Wistar rats: enhanced auditory brainstem responses are related to calretinin and nitric oxide synthase upregulation," Frontiers in Neuroanatomy, vol. 10, p. 19, 2016.

[7] S. Roy, M. M. Ryals, A. B. Van den Bruele, T. S. Fitzgerald, and L. L. Cunningham, "Sound preconditioning therapy inhibits ototoxic hearing loss in mice," The Journal of Clinical Investigation, vol. 123, no. 11, pp. 4945-4949, 2013.

[8] X. Li, Z. Zhu, W. Li, L. Wei, B. Zhao, and Z. Hao, "Polymorphism in GRHL2 gene may contribute to noise-induced hearing loss susceptibility: a meta-analysis," Brazilian Journal of Otorhinolaryngology, vol. 86, no. 3, pp. 370-375, 2020.

[9] J. Yang, J. Zhang, X. Wang et al., "Identification of functional tag single nucleotide polmorphisms within the entire CAT gene and their clinical relevance in patients with noiseinduced hearing loss," International Journal of Clinical and Experimental Pathology, vol. 8, no. 3, pp. 2852-2863, 2015. 
[10] H. Liu, G. Li, J. Lu et al., "Cellular differences in the cochlea of CBA and B6 mice may underlie their difference in susceptibility to hearing loss," Frontiers in Cellular Neuroscience, vol. 13, p. 60, 2019.

[11] B. Pouyatos, C. A. Gearhart, A. Nelson-Miller, S. Fulton, and L. D. Fechter, "Selective vulnerability of the cochlear basal turn to acrylonitrile and noise," Journal of Toxicology, vol. 2009, Article ID 908596, 2009.

[12] W. J. Clerici and L. Yang, "Direct effects of intraperilymphatic reactive oxygen species generation on cochlear function," Hearing Research, vol. 101, no. 1-2, pp. 14-22, 1996.

[13] N. Dehne, J. Lautermann, W. J. F. ten Cate, U. Rauen, and H. de Groot, "In vitro effects of hydrogen peroxide on the cochlear neurosensory epithelium of the guinea pig," Hearing Research, vol. 143, no. 1-2, pp. 162-170, 2000.

[14] S. H. Sha, R. Taylor, A. Forge, and J. Schacht, "Differential vulnerability of basal and apical hair cells is based on intrinsic susceptibility to free radicals," Hearing Research, vol. 155, no. 1-2, pp. 1-8, 2001.

[15] M. Qi, Y. Qiu, X. Zhou et al., "Regional up-regulation of NOX2 contributes to the differential vulnerability of outer hair cells to neomycin," Biochemical and Biophysical Research Communications, vol. 500, no. 2, pp. 110-116, 2018.

[16] Z. H. He, S. Y. Zou, M. Li et al., "The nuclear transcription factor FoxG1 affects the sensitivity of mimetic aging hair cells to inflammation by regulating autophagy pathways," Redox Biology, vol. 28, p. 101364, 2020.

[17] W. Liu, X. Xu, Z. Fan et al., "Wnt signaling activates TP53induced glycolysis and apoptosis regulator and protects against cisplatin-induced spiral ganglion neuron damage in the mouse cochlea," Antioxidants \& Redox Signaling, vol. 30, no. 11, pp. 1389-1410, 2019.

[18] L. Liu, Y. Chen, J. Qi et al., "Wnt activation protects against neomycin-induced hair cell damage in the mouse cochlea," Cell death \& disease, vol. 7, no. 3, p. e2136, 2016.

[19] M. E. Murphy, "The HSP70 family and cancer," Carcinogenesis, vol. 34, no. 6, pp. 1181-1188, 2013.

[20] Y. Chen, L. Li, W. Ni et al., "Bmil regulates auditory hair cell survival by maintaining redox balance," Cell death \& disease, vol. 6, no. 1, p. e1605, 2015.

[21] X. Lu, S. Sun, J. Qi et al., "Bmil regulates the proliferation of cochlear supporting cells via the canonical Wnt signaling pathway," Molecular Neurobiology, vol. 54, no. 2, pp. 1326-1339, 2017.

[22] X. Li, X. B. Mao, R. Y. Hei et al., "Protective role of hydrogen sulfide against noise-induced cochlear damage: a chronic intracochlear infusion model," PloS one, vol. 6, no. 10, p. e26728, 2011.

[23] Y. X. Wu, G. X. Zhu, X. Q. Liu et al., "Noise alters guinea pig's blood-labyrinth barrier ultrastructure and permeability along with a decrease of cochlear claudin-5 and occludin," BMC Neuroscience, vol. 15, no. 1, p. 136, 2014.

[24] X. Liu, G. Zheng, Y. Wu et al., "Lead exposure results in hearing loss and disruption of the cochlear blood-labyrinth barrier and the protective role of iron supplement," Neurotoxicology, vol. 39, pp. 173-181, 2013.

[25] M. Muller, "Frequency representation in the rat cochlea," Hearing Research, vol. 51, no. 2, pp. 247-254, 1991.

[26] Z. J. Ding, X. Chen, X. X. Tang et al., "Calpain inhibitor PD150606 attenuates glutamate induced spiral ganglion neuron apoptosis through apoptosis inducing factor pathway in vitro," PloS one, vol. 10, no. 4, p. e0123130, 2015.
[27] W. Liu, Z. Fan, Y. Han, D. Zhang, J. Li, and H. Wang, "Intranuclear localization of apoptosis-inducing factor and endonuclease $\mathrm{G}$ involves in peroxynitrite-induced apoptosis of spiral ganglion neurons," Neurological Research, vol. 34, no. 10, pp. 915-922, 2012.

[28] Z.-J. Ding, X. Chen, X.-X. Tang et al., “Apoptosis-inducing factor and calpain upregulation in glutamate-induced injury of rat spiral ganglion neurons," Molecular Medicine Reports, vol. 12, no. 2, pp. 1685-1692, 2015.

[29] C. Zhu, C. Cheng, Y. Wang et al., "Loss of ARHGEF6 causes hair cell stereocilia deficits and hearing loss in mice," Frontiers in Molecular Neuroscience, vol. 11, p. 362, 2018.

[30] Y. Wang, J. Li, X. Yao et al., "Loss of CIB2 causes profound hearing loss and abolishes mechanoelectrical transduction in mice," Frontiers in Molecular Neuroscience, vol. 10, p. 401, 2017.

[31] J. Qi, L. Zhang, F. Tan et al., "Espin distribution as revealed by super-resolution microscopy of stereocilia," American Journal of Translational Research, vol. 12, no. 1, pp. 130141, 2020.

[32] J. Qi, Y. Liu, C. Chu et al., “A cytoskeleton structure revealed by super-resolution fluorescence imaging in inner ear hair cells," Cell Discovery, vol. 5, no. 1, p. 12, 2019.

[33] S. Gao, C. Cheng, M. Wang et al., "Blebbistatin inhibits neomycin-induced apoptosis in hair cell-like HEI-OC-1 cells and in cochlear hair cells," Frontiers in Cellular Neuroscience, vol. 13, p. 590, 2020.

[34] Y. Zhang, W. Li, Z. He et al., "Pre-treatment with fasudil prevents neomycin-induced hair cell damage by reducing the accumulation of reactive oxygen species," Frontiers in Molecular Neuroscience, vol. 12, p. 264, 2019.

[35] H. Li, Y. Song, Z. He et al., "Meclofenamic acid reduces reactive oxygen species accumulation and apoptosis, inhibits excessive autophagy, and protects hair cell-like HEI-OC1 cells from cisplatin-induced damage," Frontiers in Cellular Neuroscience, vol. 12, p. 139, 2018.

[36] Z. He, L. Guo, Y. Shu et al., "Autophagy protects auditory hair cells against neomycin-induced damage," Autophagy, vol. 13, no. 11, pp. 1884-1904, 2017.

[37] Z. He, Q. Fang, H. Li et al., "The role of FOXG1 in the postnatal development and survival of mouse cochlear hair cells," Neuropharmacology, vol. 144, pp. 43-57, 2019.

[38] S. Zhang, Y. Zhang, Y. Dong et al., "Knockdown of Foxg1 in supporting cells increases the trans-differentiation of supporting cells into hair cells in the neonatal mouse cochlea," Cellular and Molecular Life Sciences, vol. 77, no. 7, pp. 1401-1419, 2020.

[39] C. Cheng, Y. Wang, L. Guo et al., "Age-related transcriptome changes in Sox $2+$ supporting cells in the mouse cochlea," Stem cell research \& therapy, vol. 10, no. 1, p. 365, 2019.

[40] D. You, L. Guo, W. Li et al., "Characterization of Wnt and Notch-responsive Lgr5+ hair cell progenitors in the striolar region of the neonatal mouse utricle," Frontiers in Molecular Neuroscience, vol. 11, p. 137, 2018.

[41] Y. Chen, X. Lu, L. Guo et al., "Hedgehog signaling promotes the proliferation and subsequent hair cell formation of progenitor cells in the neonatal mouse cochlea," Frontiers in Molecular Neuroscience, vol. 10, p. 426, 2017.

[42] Y. He, X. Lu, F. Qian, D. Liu, R. Chai, and H. Li, "Insmla is required for zebrafish posterior lateral line development," Frontiers in Molecular Neuroscience, vol. 10, p. 241, 2017. 
[43] M. Tang, J. Li, L. He et al., "Transcriptomic profiling of neural stem cell differentiation on graphene substrates," Colloids and surfaces. B, Biointerfaces, vol. 182, p. 110324, 2019.

[44] G. Li, K. Chen, D. You et al., "Laminin-coated electrospun regenerated silk fibroin mats promote neural progenitor cell proliferation, differentiation, and survival in vitro," Frontiers in Bioengineering and Biotechnology, vol. 7, p. 190, 2019.

[45] Q. Fang, Y. Zhang, X. Chen et al., “Three-dimensional graphene enhances neural stem cell proliferation through metabolic regulation," Frontiers in Bioengineering and Biotechnology, vol. 7, p. 436, 2020.

[46] S. Han, J. Sun, S. He, M. Tang, and R. Chai, "The application of graphene-based biomaterials in biomedicine," American Journal of Translational Research, vol. 11, no. 6, pp. 3246-3260, 2019.

[47] W. Yan, W. Liu, J. Qi et al., "A three-dimensional culture system with Matrigel promotes purified spiral ganglion neuron survival and function in vitro," Molecular Neurobiology, vol. 55, no. 3, pp. 2070-2084, 2018.

[48] G. Sun, W. Liu, Z. Fan et al., "The three-dimensional culture system with Matrigel and neurotrophic factors preserves the structure and function of spiral ganglion neuron in vitro," Neural Plasticity, vol. 2016, Article ID 4280407, 15 pages, 2016.

[49] S. Han, Y. Xu, J. Sun et al., "Isolation and analysis of extracellular vesicles in a morpho butterfly wing-integrated microvortex biochip," Biosensors \& Bioelectronics, vol. 154, p. 112073, 2020.

[50] B. C. Cox, R. Chai, A. Lenoir et al., "Spontaneous hair cell regeneration in the neonatal mouse cochlea in vivo," Development, vol. 141, no. 4, pp. 816-829, 2014.

[51] Y. Zhang, L. Guo, X. Lu et al., "Characterization of Lgr6+ cells as an enriched population of hair cell progenitors compared to Lgr5+ cells for hair cell generation in the neonatal mouse cochlea," Frontiers in Molecular Neuroscience, vol. 11, p. 147, 2018.

[52] S. Zhang, Y. Zhang, P. Yu et al., "Characterization of Lgr5+ progenitor cell transcriptomes after neomycin injury in the neonatal mouse cochlea," Frontiers in Molecular Neuroscience, vol. 10, p. 213, 2017.

[53] C. Cheng, L. Guo, L. Lu et al., "Characterization of the transcriptomes of Lgr5+ hair cell progenitors and Lgr5- supporting cells in the mouse cochlea," Frontiers in Molecular Neuroscience, vol. 10, p. 122, 2017.

[54] J. Wu, W. Li, C. Lin et al., "Co-regulation of the Notch and Wnt signaling pathways promotes supporting cell proliferation and hair cell regeneration in mouse utricles," Scientific Reports, vol. 6, no. 1, p. 29418, 2016.

[55] T. Wang, R. Chai, G. S. Kim et al., "Lgr5+ cells regenerate hair cells via proliferation and direct transdifferentiation in damaged neonatal mouse utricle," Nature Communications, vol. 6, no. 1, p. 6613, 2015.

[56] R. D. Kopke, J. K. M. Coleman, J. Liu, K. C. M. Campbell, and R. H. Riffenburgh, "Candidate's thesis: enhancing intrinsic cochlear stress defenses to reduce noise-induced hearing loss," The Laryngoscope, vol. 112, no. 9, pp. 1515-1532, 2002.

[57] B. Canlon and A. Fransson, "Morphological and functional preservation of the outer hair cells from noise trauma by sound conditioning," Hearing Research, vol. 84, no. 1-2, pp. 112-124, 1995.

[58] L. W. Henselman, D. Henderson, M. Subramaniam, and V. Sallustio, "The effect of 'conditioning' exposures on hearing loss from impulse noise," Hearing Research, vol. 78, no. 1, pp. 1-10, 1994.

[59] N. Yoshida and M. C. Liberman, "Sound conditioning reduces noise-induced permanent threshold shift in mice," Hearing Research, vol. 148, no. 1-2, pp. 213-219, 2000.

[60] E. B. Bertelsen, L. Chang, J. E. Gestwicki, and E. R. P. Zuiderweg, "Solution conformation of wild-type E. coli Hsp70 (DnaK) chaperone complexed with ADP and substrate," Proceedings of the National Academy of Sciences of the United States of America, vol. 106, no. 21, pp. 8471-8476, 2009.

[61] P. Bork, C. Sander, and A. Valencia, “An ATPase domain common to prokaryotic cell cycle proteins, sugar kinases, actin, and hsp70 heat shock proteins," Proceedings of the National Academy of Sciences of the United States of America, vol. 89, no. 16, pp. 7290-7294, 1992.

[62] K. M. Flaherty, C. DeLuca-Flaherty, and D. B. McKay, "Three-dimensional structure of the ATPase fragment of a $70 \mathrm{~K}$ heat-shock cognate protein," Nature, vol. 346 , no. 6285 , pp. 623-628, 1990.

[63] X. Zhu, X. Zhao, W. F. Burkholder et al., "Structural analysis of substrate binding by the molecular chaperone DnaK," Science, vol. 272, no. 5268, pp. 1606-1614, 1996.

[64] L. L. Cunningham and C. S. Brandon, "Heat shock inhibits both aminoglycoside- and cisplatin-induced sensory hair cell death," Journal of the Association for Research in Otolaryngology, vol. 7, no. 3, pp. 299-307, 2006.

[65] S. P. Francis, I. I. Kramarenko, C. S. Brandon, F. S. Lee, T. G. Baker, and L. L. Cunningham, "Celastrol inhibits aminoglycoside-induced ototoxicity via heat shock protein 32," Cell death \& disease, vol. 2, no. 8, p. e195, 2011.

[66] L. A. May, I. I. Kramarenko, C. S. Brandon et al., "Inner ear supporting cells protect hair cells by secreting HSP70," The Journal of Clinical Investigation, vol. 123, no. 8, pp. 35773587, 2013.

[67] M. Taleb, C. S. Brandon, F. S. Lee, M. I. Lomax, W. H. Dillmann, and L. L. Cunningham, "Hsp70 inhibits aminoglycosideinduced hair cell death and is necessary for the protective effect of heat shock," Journal of the Association for Research in Otolaryngology: JARO, vol. 9, no. 3, pp. 277-289, 2008.

[68] M. Taleb, C. S. Brandon, F. S. Lee, K. C. Harris, W. H. Dillmann, and L. L. Cunningham, "Hsp70 inhibits aminoglycosideinduced hearing loss and cochlear hair cell death," Cell Stress \& Chaperones, vol. 14, no. 4, pp. 427-437, 2009.

[69] M. A. Gratton, A. Eleftheriadou, J. Garcia et al., "Noiseinduced changes in gene expression in the cochleae of mice differing in their susceptibility to noise damage," Hearing Research, vol. 277, no. 1-2, pp. 211-226, 2011.

[70] H. Ninomiya, N. Ohgami, R. Oshino et al., "Increased expression level of Hsp70 in the inner ears of mice by exposure to low frequency noise," Hearing Research, vol. 363, pp. 49-54, 2018.

[71] I. Meltser and B. Canlon, "Protecting the auditory system with glucocorticoids," Hearing Research, vol. 281, no. 1-2, pp. 47$55,2011$.

[72] Y. Tahera, I. Meltser, P. Johansson, H. Salman, and B. Canlon, "Sound conditioning protects hearing by activating the hypothalamic-pituitary-adrenal axis," Neurobiology of Disease, vol. 25, no. 1, pp. 189-197, 2007.

[73] N. Yoshida, A. Kristiansen, and M. C. Liberman, "Heat stress and protection from permanent acoustic injury in mice," The Journal of Neuroscience, vol. 19, no. 22, pp. 10116-10124, 1999. 
[74] M. Yu, T. Mazor, H. Huang et al., "Direct recruitment of polycomb repressive complex 1 to chromatin by core binding transcription factors," Molecular Cell, vol. 45, no. 3, pp. 330-343, 2012.

[75] M. Abdouh, W. Chatoo, J. El Hajjar, J. David, J. Ferreira, and G. Bernier, "Bmil is down-regulated in the aging brain and displays antioxidant and protective activities in neurons," $\mathrm{PloS}$ one, vol. 7, no. 2, p. e31870, 2012.

[76] W. Chatoo, M. Abdouh, J. David et al., "The polycomb group gene Bmil regulates antioxidant defenses in neurons by repressing p53 pro-oxidant activity," The Journal of Neuroscience, vol. 29, no. 2, pp. 529-542, 2009.

[77] J. Liu, L. Cao, J. Chen et al., "Bmil regulates mitochondrial function and the DNA damage response pathway," Nature, vol. 459, no. 7245, pp. 387-392, 2009.

[78] A. Rizo, S. Olthof, L. Han, E. Vellenga, G. de Haan, and J. J. Schuringa, "Repression of BMI1 in normal and leukemic human CD34(+) cells impairs self-renewal and induces apoptosis," Blood, vol. 114, no. 8, pp. 1498-1505, 2009.

[79] S. Nakamura, M. Oshima, J. Yuan et al., "Bmil confers resistance to oxidative stress on hematopoietic stem cells," PloS one, vol. 7, no. 5, p. e36209, 2012.

[80] Q. Dong, J. E. Oh, W. Chen et al., "Radioprotective effects of Bmi-1 involve epigenetic silencing of oxidase genes and enhanced DNA repair in normal human keratinocytes," The Journal of Investigative Dermatology, vol. 131, no. 6, pp. 1216-1225, 2011.

[81] L. O. Klotz, C. Sanchez-Ramos, I. Prieto-Arroyo, P. Urbanek, H. Steinbrenner, and M. Monsalve, "Redox regulation of FoxO transcription factors," Redox Biology, vol. 6, pp. 51-72, 2015.

[82] W. He, A. Zhang, L. Qi et al., "FOXO1, a potential therapeutic target, regulates autophagic flux, oxidative stress, mitochondrial dysfunction, and apoptosis in human cholangiocarcinoma QBC939 cells," Cellular Physiology and Biochemistry, vol. 45, no. 4, pp. 1506-1514, 2018.

[83] F. Stephani, V. Scheuer, T. Eckrich et al., "Deletion of the Ca2+ channel subunit $\alpha 2 \delta 3$ differentially affects Cav2.1 and Cav2.2 currents in cultured spiral ganglion neurons before and after the onset of hearing," Frontiers in Cellular Neuroscience, vol. 13, p. 278, 2019. 